\title{
On coupled problems for viscous flow in exterior domains
}

Report

Author(s):

Feistauer, Miloslav; Schwab, Christoph

Publication date:

1996-07

Permanent link:

https://doi.org/10.3929/ethz-a-004284496

Rights / license:

In Copyright - Non-Commercial Use Permitted

Originally published in:

SAM Research Report 1996-07 


\title{
On coupled problems for viscous flow in exterior domains
}

\author{
M. Feistauer ${ }^{1}$ and C. Schwab
}

Research Report No. 96-07

July 1996

Seminar für Angewandte Mathematik

Eidgenössische Technische Hochschule

CH-8092 Zürich

Switzerland

\footnotetext{
${ }^{1}$ Charles University Prague, Faculty of Mathematics and Physics, Malostranské n. 25, 11800 Praha 1, Czech Republik, email: feist@ms.mff.cuni.cz
} 


\title{
On coupled problems for viscous flow in exterior domains
}

\author{
M. Feistauer ${ }^{1}$ and C. Schwab \\ Seminar für Angewandte Mathematik \\ Eidgenössische Technische Hochschule \\ CH-8092 Zürich \\ Switzerland
}

Research Report No. 96-07 July 1996

Dedicated to Prof. Dr. Ing. Wolfgang L. Wendland on the occasion of his 60 th birthday

\begin{abstract}
The use of the complete Navier-Stokes system in an unbounded domain is not always convenient in computations and, therefore, the Navier-Stokes problem is often truncated to a bounded domain. In this paper we simulate the interaction between the flow in this domain and the exterior flow with the aid of a coupled problem. We propose in particular a linear approximation of the exterior flow (here the Stokes flow or potential flow) coupled with the interior Navier-Stokes problem via suitable transmission conditions on the artificial interface between the interior and exterior domain. Our choice of the transmission conditions ensures the existence of a solution of the coupled problem, also for large data.
\end{abstract}

Keywords: viscous incompressible flow, Navier-Stokes equations, exterior Stokes problem, potential flow equation, transmission conditions, coupled problem, weak solution

Subject Classification: (MSC 1991): 31A05, 35A08, 45A05, 65 , 76D05.

\footnotetext{
${ }^{1}$ Charles University Prague, Faculty of Mathematics and Physics, Malostranské n. 25, 11800 Praha 1, Czech Republik, email: feist@ms.mff.cuni.cz
} 


\section{Introduction}

Many problems of fluid mechanics, continuum mechanics, heat conduction or electromagnetic fields are naturally posed in unbounded domains. Unfortunately, these formulations are not quite practical and convenient from the point of view of numerical simulation. Therefore, the unbounded domain is usually replaced by a smaller bounded computational region with an artificial boundary $\Gamma$. There is a need to prescribe suitable boundary conditions on $\Gamma$ representing the interaction of the system under consideration inside $\Gamma$ with the system in the exterior of $\Gamma$. For example, this problem is very topical in fluid dynamics in the investigation of exterior flow past bodies or obstacles. A similar problem appears in the case of flow trough ducts or pipes with artificial boundaries representing their inlets and outlets. We mention, e.g. the papers [1], [3], [14], [22], [23], [28], where a number of conditions on artificial boundaries was proposed and investigated.

Another possibility is to simulate the process in the exterior of $\Gamma$ with the aid of a suitable (preferably linear) approximation of the equations describing the process under consideration. This approach often represents the basis for the use of a combined finite element-boundary element method (FEM-BEM) for the numerical simulation of exterior problems. In the interior of an artificial interface $\Gamma$, the FEM is used for the solution of the (in general nonlinear) system describing the flow of interest whereas, provided the linear approximation in the exterior region possesses a fundamental solution, the exterior problem can be reformulated with the aid of boundary integral equations on $\Gamma$ (and solved numerically by BEM). The interior and exterior problems are coupled with the aid of suitable transmission conditions on the interface $\Gamma$.

This method has been applied by a number of authors to the modeling of various problems in unbounded domains. Let us mention, e.g. [2], [4], [7], [11], [26] which contain theory of the combined BEM and FEM, as well as applications to inviscid compressible subsonic or transonic fluid flow and elasticity or heat conductivity problems in unbounded domains.

The subject of particular interest is the simulation of viscous incompressible exterior flow described by the continuity equation and Navier-Stokes equations. In this case, the nonlinear Navier-Stokes system is used in the interior domain lying inside $\Gamma$. There are several conceivable approximations for the flow outside $\Gamma$ :

- Stokes equations,

- Oseen equations,

- inviscid Euler (or linearized Euler) equations,

- inviscid potential equation. 
The crucial problem associated with the coupled problem describing exterior viscous flow is the choice of the transmission conditions. They should be reasonable from the physical point of view and, on the other hand, there is the need to be able to prove the solvability of the coupled problem. For example, in [13] the existence of a solution to a coupled "interior Navier-Stokes - exterior Stokes" problem is guaranteed by the a priori assumption that the normal component of the velocity vanishes on the whole interface $\Gamma$, which is not quite reasonable from the physical point of view.

In [24], the coupled "Stokes-Stokes" problem was treated together with the continuity of the velocity as well as normal stress on $\Gamma$. The same transmission conditions were used in [15], [16] for the coupling "interior Navier-Stokes - exterior Stokes" and "interior Navier-Stokes - exterior Oseen". However, the mentioned transmission conditions allow to prove the existence of the solution only for small data (small Reynolds number and/or small outer volume force). Moreover, the results formulated in [15], [16] are true only for zero farfield velocity, as can be found by careful reading. In addition, the analysis carried out in [15], [16] is restricted to small data because of missing coercivity (a similar difficulty appears in [14] for the pipe or duct flow with a "natural" outlet condition).

The mathematical analysis of the coupling of the interior Navier-Stokes problem with the exterior Stokes problem or exterior potential problem is the purpose of the present paper. In both cases we propose new transmission conditions on the artificial interface. Under these conditions we obtain in each case a coercive, nonlinear coupled problem and prove its solvability for arbitrarily large data. In the case of potential flow, however, we exhibit some pathological examples which indicate that this model is overly simplicistic and its inherent modelling error is too large. If the Poincaré-Steklov operator corresponding to the linear, exterior problem is realized by boundary integral operators, the results of the paper represent a theoretical basis for the numerical solution of the exterior coupled problem with the aid of the combined FEM and BEM.

\section{Formulation of the problem}

Let $\Omega^{\prime} \subset \mathbb{R}^{3}$ be an unbounded domain whose complement $\Omega=\mathbb{R}^{3}-\bar{\Omega}^{\prime}(\bar{M}$ denotes the closure of a set $M \subset \mathbb{R}^{3}$ ) consists of a finite number of bounded domains $\Omega_{i}$, $i=1, \ldots, k$, with mutually disjoint and sufficiently smooth boundaries $\partial \Omega_{i}$. Then $\Gamma_{0}:=\partial \Omega^{\prime}=\bigcup_{i=1}^{k} \partial \Omega_{i}$.

We consider incompressible flow in the exterior domain $\Omega^{\prime}$ past impermeable bodies or obstacles $\Omega_{1}, \ldots, \Omega_{k}$. Assuming that the flow is homogeneous far away 
from the bodies, we can introduce the following classical formulation of the flow problem:

Find $\boldsymbol{u}=\left(u_{1}, u_{2}, u_{3}\right): \overline{\Omega^{\prime}} \rightarrow \mathbb{R}^{3}$ and $p: \bar{\Omega}^{\prime} \rightarrow \mathbb{R}$ such that

$$
\begin{aligned}
& u_{i} \in C^{2}\left(\bar{\Omega}^{\prime}\right), \quad i=1,2,3, p \in C^{1}\left(\bar{\Omega}^{\prime}\right), \\
& -\nu \Delta \boldsymbol{u}+(\boldsymbol{u} \cdot \nabla) \boldsymbol{u}+\nabla p=\boldsymbol{f} \text { in } \Omega^{\prime}, \\
& \operatorname{div} \boldsymbol{u}=0 \text { in } \Omega^{\prime}, \\
& \left.\boldsymbol{u}\right|_{\Gamma_{0}}=0 \\
& \lim _{|x| \rightarrow \infty} \boldsymbol{u}(x)=\boldsymbol{u}_{\infty} .
\end{aligned}
$$

We use the standard notation: $x=\left(x_{1}, x_{2}, x_{3}\right) \in \mathbb{R}^{3}$ denotes a point of the 3-dimensional Euclidean space with Cartesian coordinates $x_{i}(i=1,2,3), \boldsymbol{u}=$ $\left(u_{1}, u_{2}, u_{3}\right)$ is the velocity vector with components $u_{i}$ in the directions $x_{i}, \boldsymbol{f}$ the density of outer volume force, $\nu>0$ the kinematic viscosity, $p$ the kinematic static pressure, $\boldsymbol{u}_{\infty}=\left(u_{\infty 1}, u_{\infty 2}, u_{\infty 3}\right)$ the velocity of the farfield, $\nabla=\left(\partial / \partial x_{1}, \partial / \partial x_{2}, \partial / \partial x_{3}\right)$, $(1.1 \mathrm{~b})$ and $(1.1 \mathrm{c})$ are the Navier-Stokes equations and the continuity equation, respectively, and $\nu, \boldsymbol{u}_{\infty} \neq 0, \boldsymbol{f}$ are given data. Let us assume that $\boldsymbol{f}$ has compact support, i.e. supp $\boldsymbol{f} \subset \Omega^{\prime} \cup \Gamma_{0}$. Then it is classical (see [10], Chapter IX and the references there) that $(1.1 \mathrm{~b}-1.1 \mathrm{e})$ admits at least one solution $\boldsymbol{u}$ with finite Dirichlet integral, i.e.

$$
\int_{\Omega^{\prime}} \nabla \boldsymbol{u}: \nabla \boldsymbol{u} d x \leq M .
$$

Moreover, this solution satisfies ([10], Remark IX.8.1)

$$
\boldsymbol{u}(x)-\boldsymbol{u}_{\infty}=O\left(|x|^{-1}\right) \quad|x| \rightarrow \infty
$$

Since the above formulation in the unbounded domain $\Omega^{\prime}$ is not convenient for numerical discretization, as was stated in Introduction, we introduce an artificial interface $\Gamma \subset \Omega^{\prime}$, dividing $\Omega^{\prime}$ into two subdomains: a bounded interior domain $\Omega^{-}$ with $\partial \Omega^{-}=\Gamma_{0} \cup \Gamma$, in which we consider the Navier-Stokes system $(1.1 \mathrm{~b})-(1.1 \mathrm{c})$, and an unbounded domain $\Omega^{+}$lying outside $\Gamma$, with $\partial \Omega^{+}=\Gamma$ and $\bar{\Omega}^{+}=\Omega^{+} \cup \Gamma$, see Figure 1. 


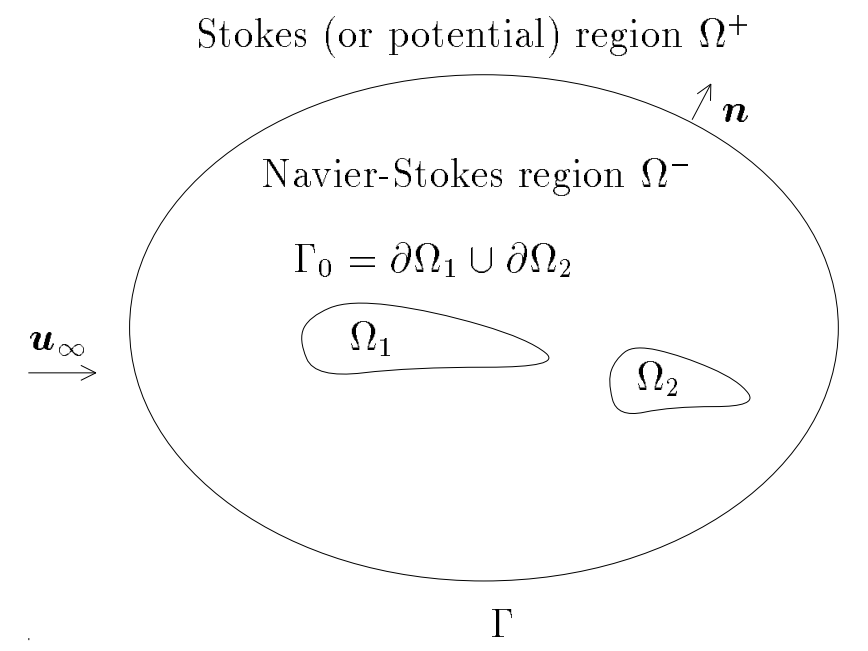

Fig. 1

Let supp $f \subset \Omega^{-} \cup \Gamma_{0}$ and let $\partial \Omega^{-}$be sufficiently regular. In what follows it will be enough to suppose that $\partial \Omega^{-}=\Gamma_{0} \cup \Gamma$ is strongly local Lipschitz-continuous (cf. [17] or [6], Par. 1.2.27). In $\Omega^{+}$we use some of the flow approximations mentioned in the Introduction. Here we will be mainly concerned with Stokes flow in $\Omega^{+}$but in Section 6 we discuss briefly the coupled "Navier-Stokes-inviscid potential" problem.

An important question is the choice of coupling transmission conditions on T. In [24] the coupling "Stokes-Stokes" was treated via the transmission conditions requiring the continuity of the velocity and normal stress across $\Gamma$. According to [1], the dynamic pressure $p+|\boldsymbol{u}|^{2} / 2$ plays the same role for the Navier-Stokes equations as the kinematic static pressure $p$ for the Stokes problem. This is the motivation for our choice of the transmission conditions in the following classical formulation of the coupled problem:

Find $\boldsymbol{u}^{ \pm}=\left(u_{1}^{ \pm}, u_{2}^{ \pm}, u_{3}^{ \pm}\right): \bar{\Omega}^{ \pm} \rightarrow \mathbb{R}^{3}, p^{ \pm}: \bar{\Omega}^{ \pm} \rightarrow \mathbb{R}$ such that

$$
\begin{aligned}
& u_{i}^{ \pm} \in C^{2}\left(\bar{\Omega}^{ \pm}\right), \quad i=1,2,3, \quad p^{ \pm} \in C^{1}\left(\bar{\Omega}^{ \pm}\right), \\
& -\nu \Delta \boldsymbol{u}^{-}+\left(\boldsymbol{u}^{-} \cdot \nabla\right) \boldsymbol{u}^{-}+\nabla p^{-}=\boldsymbol{f} \text { in } \Omega^{-}, \\
& \operatorname{div} \boldsymbol{u}^{-}=0 \text { in } \Omega^{-}, \\
& \left.\boldsymbol{u}^{-}\right|_{\Gamma_{0}}=0, \\
& -\nu \Delta \boldsymbol{u}^{+}+\nabla p^{+}=0 \text { in } \Omega^{+},
\end{aligned}
$$




$$
\begin{aligned}
& \operatorname{div} \boldsymbol{u}^{+}=0 \text { in } \Omega^{+}, \\
& \lim _{|x| \rightarrow \infty} \boldsymbol{u}^{+}(x)=\boldsymbol{u}_{\infty}, \\
& \boldsymbol{u}^{-}=\boldsymbol{u}^{+} \text {on } \Gamma, \\
& -\left(p^{-}+\frac{1}{2}\left|\boldsymbol{u}^{-}\right|^{2}\right) \boldsymbol{n}+2 \nu \operatorname{DD}\left(\boldsymbol{u}^{-}\right) \boldsymbol{n}=-\lambda\left(\boldsymbol{u}^{+}, p^{+}\right) \text {on } \Gamma .
\end{aligned}
$$

Remark 1.1 The selection of $(1.3 \mathrm{e}-1.3 \mathrm{~g})$ in $\Omega^{+}$is motivated by the fact that the velocity field $\boldsymbol{u}^{+}$has, as solution of a Stokes problem in $\Omega^{+}$, also the velocity asymptotics (1.2) in the farfield, provided, however, $\Gamma$ is sufficiently smooth (cf. [10], Theorem V.3.2), and under the provision that a solution of the coupled problem (1.3) exists, of course. This also indicates that increasing the size of $\Omega^{-}$resp. $\Gamma$ will reduce the modeling error introduced by adopting $(1.3 \mathrm{e}-1.3 \mathrm{~g})$ in $\Omega^{+}$. Note also that (1.3) cannot represent in $\Omega^{+}$the parabolic wake trailing $\Omega$. In order to do this, one should use Oseen flow in $\Omega^{+}$. For these issues as well as for modeling error estimates, we refer to [8].

The functions $\boldsymbol{u}^{-}, p^{-}$and $\boldsymbol{u}^{+}, p^{+}$satisfying (1.3) are called a classical solution of the coupled problem. Here $\boldsymbol{n}$ denotes the unit outer normal to $\partial \Omega^{-}$(i.e. $\boldsymbol{n}$ points from $\Omega^{-}$into $\Omega^{+}$on $\Gamma$, see Figure 1$)$ and $\operatorname{DD}(\boldsymbol{u})$ is the velocity deformation tensor:

$$
\mathbb{D}(\boldsymbol{u})=\left(D_{i j}(\boldsymbol{u})\right)_{i, j=1}^{3}, D_{i j}(\boldsymbol{u})=\frac{1}{2}\left(\frac{\partial u_{i}}{\partial x_{j}}+\frac{\partial u_{j}}{\partial x_{i}}\right) .
$$

Further, we set

$$
\begin{aligned}
\sigma_{i j}(\boldsymbol{u}, p) & =-p \delta_{i j}+2 \nu D_{i j}(\boldsymbol{u}) \\
\sigma_{\mathbf{n}}(\boldsymbol{u}, p) & =\left(\sum_{j=1}^{3} \sigma_{i j}(\boldsymbol{u}, p) n_{j}\right)_{i=1}^{3}=-p \boldsymbol{n}+2 \nu \mathbb{D}(\boldsymbol{u}) \boldsymbol{n}
\end{aligned}
$$

i.e. $\sigma_{i j}(\boldsymbol{u}, p)$ and $\sigma_{\mathbf{n}}(\boldsymbol{u}, p)$ are the components of the stress tensor and the projection of the stress tensor into the direction $\boldsymbol{n}$, respectively. By $\lambda\left(\boldsymbol{u}^{+}, p^{+}\right)$in (1.3i) we denote a suitable boundary operator with values in $\mathbb{R}^{3}$. With respect to $(1.5)$, condition (1.3i) can be written as

$$
\sigma_{\mathbf{n}}\left(\boldsymbol{u}^{-}, p^{-}\right)-\frac{1}{2}\left|\boldsymbol{u}^{-}\right|^{2} \boldsymbol{n}=-\lambda\left(\boldsymbol{u}^{+}, p^{+}\right) .
$$

It represents a general transmission condition suitable for coupled problems modeling viscous flow. The operator $\lambda\left(\boldsymbol{u}^{+}, p^{+}\right)$must be specified according to the character of the flow model in $\Omega^{+}$. Its choice is governed by the following requirements: 
1) It is possible to prove the existence of a (weak) solution to the coupled problem under conditions similar to those ensuring the existence of a solution to the original, nonlinear problem on the unbounded domain.

2) The operator $\lambda$ is compatible with the exterior ansatz. As we shall see in Section 5, in the case of the exterior Stokes flow, it is suitable to set $\lambda\left(\boldsymbol{u}^{+}, p^{+}\right)=$ $-\sigma_{\mathbf{n}}\left(\boldsymbol{u}^{+}, p^{+}\right)$on $\Gamma$ (in the distributional sense).

Let us finally note that if $\boldsymbol{u}=\left(u_{1}, u_{2}, u_{3}\right), u_{i} \in C^{2}\left(\Omega^{ \pm}\right), i=1,2,3, \operatorname{div} \boldsymbol{u}=0$ in $\Omega^{ \pm}$, then

$$
\nu \Delta u_{i}=2 \nu \sum_{j=1}^{3} \frac{\partial D_{i j}(\boldsymbol{u})}{\partial x_{j}} \text { in } \Omega^{ \pm}
$$

\section{Function spaces}

In order to reformulate the problem (1.3) in a weak sense, we introduce some function spaces (for details see, e.g. [17]). The symbols $C^{\infty}\left(\Omega^{-}\right)$and $C^{\infty}\left(\bar{\Omega}^{-}\right)$denote the spaces of infinitely differentiable functions in $\Omega^{-}$and $\bar{\Omega}^{-}$, respectively. Further, we set $C_{0}^{\infty}\left(\Omega^{-}\right)=\left\{v \in C^{\infty}\left(\bar{\Omega}^{-}\right) ; \operatorname{supp} v \subset \Omega^{-}\right\}$and $C_{0}^{\infty}\left(\Omega^{-} \cup \Gamma\right)=\left\{v \in C^{\infty}\left(\bar{\Omega}^{-}\right)\right.$; $\left.\operatorname{supp} v \subset \Omega^{-} \cup \Gamma\right\}(\operatorname{supp} v$ denotes the support of $v)$. Similarly we define the spaces $C^{\infty}\left(\Omega^{+}\right), C^{\infty}\left(\bar{\Omega}^{+}\right)$and set $C_{0}^{\infty}\left(\Omega^{+}\right)=\left\{v \in C^{\infty}\left(\bar{\Omega}^{+}\right)\right.$; supp $v \subset \Omega^{+}$is bounded $\}$.

By $L^{2}\left(\Omega^{ \pm}\right)$we denote the Lebesgue space of square integrable functions over $\Omega^{ \pm}$. The norm in $L^{2}\left(\Omega^{ \pm}\right)$will be denoted by $\|\cdot\|_{0, \Omega^{ \pm}}$. Similarly we define the space $L^{2}(\Gamma)$ and denote its norm by $\|\cdot\|_{0, \Gamma}$.

By $H^{1}\left(\Omega^{-}\right)$we denote the Sobolev space $W^{1,2}\left(\Omega^{-}\right)$equipped with the norm

$$
\|u\|_{1, \Omega^{-}}=\left(\int_{\Omega^{-}}\left(|u|^{2}+|\nabla u|^{2}\right) d x\right)^{1 / 2}, \quad u \in H^{1}\left(\Omega^{-}\right) .
$$

The trace of a function $u \in H^{1}\left(\Omega^{-}\right)$on $\Gamma$ will be denoted by $\gamma_{0} u$. Hence, $\gamma_{0}$ : $H^{1}\left(\Omega^{-}\right) \rightarrow L^{2}(\Gamma)$. We put $H_{0}^{1}\left(\Omega^{-}\right)=$closure of $C_{0}^{\infty}\left(\Omega^{-}\right)$in $H^{1}\left(\Omega^{-}\right)=\{v \in$ $\left.H^{1}\left(\Omega^{-}\right) ; v_{\mid \partial \Omega^{-}}=0\right\}$. Further, we will work with the Sobolev-Slobodetskii space $H^{1 / 2}(\Gamma)=W^{1 / 2,2}(\Gamma)$ (cf. [17], Par. 6.8), which can be characterized as

$$
H^{1 / 2}(\Gamma)=\left\{\gamma_{0} u ; u \in H^{1}\left(\Omega^{-}\right)\right\}
$$

The norm in $H^{1 / 2}(\Gamma)$ will be denoted by $\|\cdot\|_{1 / 2, \Gamma}$. The symbol $H^{-1 / 2}(\Gamma)$ will denote the dual of $H^{1 / 2}(\Gamma)$. In $H^{-1 / 2}(\Gamma)$ we define the norm by

$$
\|\varphi\|_{-1 / 2, \Gamma}=\sup _{0 \neq v \in H^{1 / 2}(\Gamma)} \frac{\langle\varphi, v\rangle}{\|v\|_{1 / 2, \Gamma}}, \varphi \in H^{-1 / 2}(\Gamma),
$$


where $\langle\cdot, \cdot\rangle$ is the duality pairing between $H^{-1 / 2}(\Gamma)$ and $H^{1 / 2}(\Gamma)$ induced by the $L^{2}(\Gamma)$-scalar product. This means that $\langle\varphi, v\rangle$ is the value of the functional $\varphi \in$ $H^{-1 / 2}(\Gamma)$ at the point $v \in H^{1 / 2}(\Gamma)$ and

$$
\langle\varphi, v\rangle=\int_{\Gamma} \varphi v d S \text { for } \varphi \in L^{2}(\Gamma), v \in H^{1 / 2}(\Gamma) .
$$

$H^{1 / 2}(\Gamma)$ is a reflexive, separable Banach space.

In order to analyze the problem in $\Omega^{+}$, we define the weighted Sobolev space

$$
W^{1}\left(\Omega^{+}\right)=\left\{u ;\left(1+|x|^{2}\right)^{-1 / 2} u \in L^{2}\left(\Omega^{+}\right), \frac{\partial u}{\partial x_{i}} \in L^{2}\left(\Omega^{+}\right), \quad i=1,2,3\right\},
$$

equipped with the norm

$$
\|u\|_{1, \Omega^{+}}=\left\{\int_{\Omega^{+}}\left(1+|x|^{2}\right)^{-1}|u(x)|^{2} d x+|u|_{1, \Omega^{+}}^{2}\right\}^{1 / 2},
$$

where the seminorm $|\cdot|_{1, \Omega}+$ reads

$$
|u|_{1, \Omega^{+}}=\left(\int_{\Omega^{+}}|\nabla u|^{2} d x\right)^{1 / 2} .
$$

By [5], Theorem 1 , page $118,|\cdot|_{1, \Omega^{+}}$is a norm on $W^{1}\left(\Omega^{+}\right)$equivalent to the norm $\|\cdot\|_{1, \Omega+}$.

The space $H^{1 / 2}(\Gamma)$ can be interpreted as the space of traces of all $u \in W^{1}\left(\Omega^{+}\right)$. The trace of $u \in W^{1}\left(\Omega^{+}\right)$on $\Gamma$ will be again denoted by $\gamma_{0} u$. In what follows, it will be convenient to use in $H^{1 / 2}(\Gamma)$ the norm

$$
\left\|u_{0}\right\|_{1 / 2, \Gamma}=\inf _{\substack{u \in W^{1}\left(\Omega^{+}\right) \\ \gamma_{0} u=u_{0}}}\|u\|_{1, \Omega^{+}}, \quad u_{0} \in H^{1 / 2}(\Gamma)
$$

equivalent to the Sobolev-Slobodetskii norm defined in $H^{1 / 2}(\Gamma)$ (cf. [17], Theorems $6.8 .13,6.9 .2)$.

Further, we put

$$
W_{0}^{1}\left(\Omega^{+}\right)=\text {closure of } C_{0}^{\infty}\left(\Omega^{+}\right) \text {in } W^{1}\left(\Omega^{+}\right) \text {. }
$$

With the aid of the partition of unity and regularization we can show that

$$
W_{0}^{1}\left(\Omega^{+}\right)=\left\{v \in W^{1}\left(\Omega^{+}\right) ; \gamma_{0} v=0 \text { on } \Gamma\right\} \text {. }
$$


In the sequel we will work with 3 -dimensional vector valued functions. To this end, for a Banach space $X$ with a norm $\|\cdot\|$ we define the space $\boldsymbol{X}=X^{3}=$ $X \times X \times X=\left\{\boldsymbol{u}=\left(u_{1}, u_{2}, u_{3}\right) ; u_{i} \in X, i=1,2,3\right\}$ equipped with the norm

$$
\|\boldsymbol{u}\|=\left(\sum_{i=1}^{3}\left\|u_{i}\right\|^{2}\right)^{1 / 2}, \quad \boldsymbol{u}=\left(u_{1}, u_{2}, u_{3}\right) \in \boldsymbol{X}
$$

In this way we introduce the spaces $\boldsymbol{L}^{2}\left(\Omega^{ \pm}\right), \boldsymbol{L}^{2}(\Gamma), \boldsymbol{H}^{1}\left(\Omega^{-}\right), \boldsymbol{W}^{1}\left(\Omega^{+}\right), \boldsymbol{H}^{1 / 2}(\Gamma)$ etc. equipped with norms defined by $(2.10)$ and denoted by $\|\cdot\|_{0, \Omega^{ \pm}},\|\cdot\|\left\|_{0, \Gamma},\right\| \cdot \|_{1, \Omega^{-}}$, $\|\cdot\|_{1, \Omega^{+}},\|\cdot\|_{1 / 2, \Gamma}$ etc. The scalar product in $\boldsymbol{L}^{2}\left(\Omega^{-}\right)$is defined as

$$
\begin{aligned}
& (\boldsymbol{u}, \boldsymbol{v})_{0, \Omega^{-}}=\int_{\Omega^{-}} \sum_{i=1}^{3} u_{i} v_{i} d x, \\
& \boldsymbol{u}=\left(u_{1}, u_{2}, u_{3}\right), \boldsymbol{v}=\left(v_{1}, v_{2}, v_{3}\right) \in \boldsymbol{L}^{2}\left(\Omega^{-}\right)
\end{aligned}
$$

(similarly for $\boldsymbol{L}^{2}\left(\Omega^{+}\right)$and $\boldsymbol{L}^{2}(\Gamma)$ ).

Now let us define subspaces of $\boldsymbol{H}^{1}\left(\Omega^{-}\right)$and $\boldsymbol{W}^{1}\left(\Omega^{+}\right)$associated with the analysis of viscous flow in $\Omega^{-}$and $\Omega^{+}$:

$$
\begin{aligned}
\mathcal{V}\left(\Omega^{-}\right) & =\left\{\boldsymbol{v} \in \boldsymbol{C}_{0}^{\infty}\left(\Omega^{-} \cup \Gamma\right): \operatorname{div} \boldsymbol{v}=0 \text { in } \Omega^{-}\right\} \\
\boldsymbol{V}\left(\Omega^{-}\right) & =\text {closure of } \mathcal{V}\left(\Omega^{-}\right) \text {in } \boldsymbol{H}^{1}\left(\Omega^{-}\right), \\
\boldsymbol{V}_{1}\left(\Omega^{-}\right) & =\left\{\boldsymbol{v} \in \boldsymbol{H}^{1}\left(\Omega^{-}\right): \boldsymbol{v}_{\mid \Gamma_{0}}=0, \operatorname{div} \boldsymbol{v}=0 \text { in } \Omega^{-}\right\} .
\end{aligned}
$$

Let us notice that

$$
\int_{\Gamma} \boldsymbol{v} \cdot \boldsymbol{n} d S=0 \quad \forall \boldsymbol{v} \in \boldsymbol{V}_{1}\left(\Omega^{-}\right) .
$$

Lemma 2.1 We have $V_{1}\left(\Omega^{-}\right)=V\left(\Omega^{-}\right)$.

\section{Proof:}

a) Obviously, $\boldsymbol{V}:=\boldsymbol{V}\left(\Omega^{-}\right) \subset \boldsymbol{V}_{1}:=\boldsymbol{V}_{1}\left(\Omega^{-}\right)$.

b) Let us prove that $\boldsymbol{V}_{1} \subset \boldsymbol{V}$. Pick $\boldsymbol{v} \in \boldsymbol{V}_{1}$. Then $\boldsymbol{g}:=\gamma_{0} \boldsymbol{v}=\left.\boldsymbol{v}\right|_{\Gamma} \in \boldsymbol{H}^{1 / 2}(\Gamma)$ and, by $(2.15)$,

$$
\int_{\Gamma} \boldsymbol{g} \cdot \boldsymbol{n} d S=0 .
$$

Consider a bounded domain $\Omega^{*} \subset \Omega^{+}$with $\partial \Omega^{*}=\Gamma \cup \Gamma^{*}$, where $\Gamma^{*}$ is a sufficiently large sphere and $\Gamma$ lies in the interior of $\Gamma^{*}$. Let us set $\boldsymbol{g}_{\mid \Gamma^{*}}=0$. Then, by [12], 
Lemma 2.2 , page 24 , there exists $\boldsymbol{v}^{*} \in \boldsymbol{H}^{1}\left(\Omega^{*}\right)$ such that $\boldsymbol{v}_{\mid \partial \Omega^{*}}^{*}=\boldsymbol{g}$ and $\operatorname{div} \boldsymbol{v}^{*}=0$ in $\Omega^{*}$. Hence, $\gamma_{0} \boldsymbol{v}^{*}=\gamma_{0} \boldsymbol{v}$ on $\Gamma$.

For the bounded domain $\widetilde{\Omega}=\Omega^{-} \cup \Omega^{*} \cup \Gamma$ we define

$$
\begin{aligned}
\mathcal{V}(\tilde{\Omega}) & =\left\{\boldsymbol{v} \in \boldsymbol{C}_{0}^{\infty}(\widetilde{\Omega}) ; \operatorname{div} \boldsymbol{v}=0 \text { in } \tilde{\Omega}\right\} \\
\boldsymbol{V}_{1}(\widetilde{\Omega}) & =\left\{\boldsymbol{v} \in \boldsymbol{H}_{0}^{1}(\widetilde{\Omega}) ; \operatorname{div} \boldsymbol{v}=0 \text { in } \widetilde{\Omega}\right\} .
\end{aligned}
$$

By [12], Corollary 2.5, page 26 , the space $\mathcal{V}(\widetilde{\Omega})$ is dense in $\boldsymbol{V}_{1}(\widetilde{\Omega})$.

If we define $\boldsymbol{w}: \widetilde{\Omega} \rightarrow \mathbb{R}^{3}$ so that

$$
\left.\boldsymbol{w}\right|_{\Omega^{-}}=\boldsymbol{v},\left.\quad \boldsymbol{w}\right|_{\Omega^{*}}=v^{*}
$$

then $\boldsymbol{w} \in \boldsymbol{V}_{1}(\tilde{\Omega})$. By the density of $\mathcal{V}(\tilde{\Omega})$ in $\boldsymbol{V}_{1}(\tilde{\Omega})$, there exists a sequence $\boldsymbol{w}^{n} \in$ $\mathcal{V}(\widetilde{\Omega})$ such that

$$
\left\|\boldsymbol{w}^{n}-\boldsymbol{w}\right\|_{1, \widetilde{\Omega}} \rightarrow 0 \text { as } n \rightarrow \infty
$$

Now we put $\boldsymbol{v}^{n}=\left.\boldsymbol{w}^{n}\right|_{\Omega^{-}} \in \mathcal{V}\left(\Omega^{-}\right)$. Then (2.16) implies that

$$
\left\|\boldsymbol{v}^{n}-\boldsymbol{v}\right\|_{1, \Omega^{-}} \rightarrow 0 \text { as } n \rightarrow \infty
$$

which means $\boldsymbol{v} \in \boldsymbol{V}$. Hence $\boldsymbol{V}_{1} \subset \boldsymbol{V}$.

With respect to $(2.15)$, we set

$$
\boldsymbol{H}_{0}^{1 / 2}(\Gamma)=\left\{\boldsymbol{v} \in \boldsymbol{H}^{1 / 2}(\Gamma) ; \int_{\Gamma} \boldsymbol{v} \cdot \boldsymbol{n} d S=0\right\},
$$

which is a closed subspace of $\boldsymbol{H}^{1 / 2}(\Gamma)$.

In analogy to $(2.12)-(2.14)$ we introduce the following spaces:

$$
\begin{aligned}
& \boldsymbol{W}\left(\Omega^{+}\right)=\left\{\boldsymbol{v} \in \boldsymbol{W}^{1}\left(\Omega^{+}\right) ; \operatorname{div} \boldsymbol{v}=0 \text { in } \Omega^{+}\right\} \\
& \mathcal{V}_{0}\left(\Omega^{+}\right)=\left\{\boldsymbol{v} \in \boldsymbol{C}_{0}^{\infty}\left(\Omega^{+}\right) ; \operatorname{div} \boldsymbol{v}=0 \text { in } \Omega^{+}\right\} \\
& \boldsymbol{V}_{0}\left(\Omega^{+}\right)=\text {closure of } \mathcal{V}_{0}\left(\Omega^{+}\right) \text {in } \boldsymbol{W}^{1}\left(\Omega^{+}\right) \\
& \boldsymbol{V}_{01}\left(\Omega^{+}\right)=\boldsymbol{W}\left(\Omega^{+}\right) \cap \boldsymbol{W}_{0}^{1}\left(\Omega^{+}\right) .
\end{aligned}
$$

It is possible to show that $\gamma_{0} \boldsymbol{v}=0$ on $\Gamma$ for $\boldsymbol{v} \in \boldsymbol{V}_{01}\left(\Omega^{+}\right)$or $\boldsymbol{v} \in \boldsymbol{V}_{0}\left(\Omega^{+}\right)$.

Comparing our definitions with [10], Volume I, Par. II.5 (Theorem 5.1) and Par. III.5, we find that the spaces $\boldsymbol{W}^{1}\left(\Omega^{+}\right), \boldsymbol{W}_{0}^{1}\left(\Omega^{+}\right), \boldsymbol{V}_{01}\left(\Omega^{+}\right)$and $\boldsymbol{V}_{0}\left(\Omega^{+}\right)$can be identified with Galdi's spaces $D^{1,2}\left(\Omega^{+}\right), D_{0}^{1,2}\left(\Omega^{+}\right), \hat{\mathcal{D}}_{0}^{1,2}\left(\Omega^{+}\right)$and $\mathcal{D}_{0}^{1,2}\left(\Omega^{+}\right)$, respectively. Then, by [10], Volume I, Par. III.5, page 160, we have 
Lemma 2.2 It holds

$$
V_{01}\left(\Omega^{+}\right)=V_{0}\left(\Omega^{+}\right)
$$

Lemma 2.3 For any $\boldsymbol{u}_{0} \in \boldsymbol{H}_{0}^{1 / 2}(\Gamma)$ there exists an extension $\boldsymbol{R} \boldsymbol{u}_{0} \in \boldsymbol{W}\left(\Omega^{+}\right)$such that $\gamma_{0}\left(\boldsymbol{R} \boldsymbol{u}_{0}\right)=\boldsymbol{u}_{0}$. Moreover, there exists $c_{1}>0$ such that

$$
\inf _{\mathbf{v} \in \mathbf{V}_{0}\left(\Omega^{+}\right)}\left\|\boldsymbol{R} \boldsymbol{u}_{0}+\boldsymbol{v}\right\|_{1, \Omega^{+}} \leq c_{1}\left\|\boldsymbol{u}_{0}\right\|_{1 / 2, \Gamma} \quad \forall \boldsymbol{u}_{0} \in \boldsymbol{H}_{0}^{1 / 2}(\Gamma) .
$$

Proof: Let $\Omega^{*}$ be defined in the same way as in the proof of Lemma 2.1. Then $\partial \Omega^{*}=\Gamma \cup \Gamma^{*}, \Gamma^{*} \subset \Omega^{+}$. We define $\boldsymbol{g}: \partial \Omega^{*} \rightarrow \mathbb{R}^{3}$ so that

$$
\left.\boldsymbol{g}\right|_{\Gamma}=\boldsymbol{u}_{0} \in \boldsymbol{H}_{0}^{1 / 2}(\Gamma),\left.\boldsymbol{g}\right|_{\Gamma^{*}}=0 \text {. }
$$

In virtue of [12], Lemma 2.2, page 24, there exists $\boldsymbol{R}^{*} \boldsymbol{u}_{0} \in \boldsymbol{H}^{1}\left(\Omega^{*}\right)$ such that $\boldsymbol{R}^{*} \boldsymbol{u}_{0} \mid \partial \Omega^{*}=\boldsymbol{g}, \operatorname{div}\left(\boldsymbol{R}^{*} \boldsymbol{u}_{0}\right)=0$ in $\Omega^{*}$ and

$$
\inf \left\{\left\|\boldsymbol{R}^{*} \boldsymbol{u}_{0}+\boldsymbol{v}^{*}\right\|_{1, \Omega^{*}} ; \boldsymbol{v}^{*} \in \boldsymbol{H}_{0}^{1}\left(\Omega^{*}\right), \operatorname{div} \boldsymbol{v}^{*}=0 \text { in } \Omega^{*}\right\} \leq c_{1}\left\|\boldsymbol{u}_{0}\right\|_{1 / 2, \Gamma}
$$

with $c_{1}$ independent of $\boldsymbol{u}_{0}$. Denoting by $\boldsymbol{R} \boldsymbol{u}_{0}$ and $\boldsymbol{v}$ the extension of $\boldsymbol{R}^{*} \boldsymbol{u}_{0}$ and $\boldsymbol{v}^{*}$ by zero outside $\Gamma^{*}$, we see that $\boldsymbol{R} \boldsymbol{u}_{0} \in \boldsymbol{W}\left(\Omega^{+}\right)$is the sought function, and that $\boldsymbol{v} \in \boldsymbol{V}_{0}\left(\Omega^{+}\right)$and

$$
\begin{aligned}
& \inf _{\boldsymbol{v} \in \mathbf{V}_{0}\left(\Omega^{+}\right)}\left\|\boldsymbol{R} \boldsymbol{u}_{0}+\boldsymbol{v}\right\|_{1, \Omega^{+}} \leq \\
& \leq \inf \left\{\left\|\boldsymbol{R} \boldsymbol{u}_{0}+\boldsymbol{v}\right\|_{1, \Omega^{+}} ; \boldsymbol{v} \in \boldsymbol{V}_{0}\left(\Omega^{+}\right), \boldsymbol{v}=0 \text { outside } \Gamma^{*}\right\} \\
& =\inf \left\{\left\|\boldsymbol{R}^{*} \boldsymbol{u}_{0}+\boldsymbol{v}^{*}\right\|_{1, \Omega^{*}} ; \boldsymbol{v}^{*} \in \boldsymbol{H}_{0}^{1}\left(\Omega^{*}\right), \operatorname{div} \boldsymbol{v}^{*}=0 \text { in } \Omega^{*}\right\} \\
& \leq c_{1}\left\|\boldsymbol{u}_{0}\right\|_{1 / 2, \Gamma} .
\end{aligned}
$$

\section{Weak formulation}

Let $\left(\boldsymbol{u}^{-}, p^{-}\right)$and $\left(\boldsymbol{u}^{+}, p^{+}\right)$satisfy (1.3a - i). Multiplying (1.3b) rewritten with the aid of (1.6) by an arbitrary $\boldsymbol{v} \in \mathcal{V}\left(\Omega^{-}\right)$, integrating over $\Omega^{-}$, applying Green's theorem and using (1.3i) and the relations $\operatorname{div} \boldsymbol{v}=0$ in $\Omega^{-}$and $\left.\boldsymbol{v}\right|_{\Gamma_{0}}=0$, we obtain the identity

$$
\begin{aligned}
& 2 \nu \int_{\Omega^{-}} \sum_{i, j=1}^{3} D_{i j}\left(\boldsymbol{u}^{-}\right) D_{i j}(\boldsymbol{v}) d x+\int_{\Omega^{-}} \sum_{i, j=1}^{3} u_{j}^{-} \frac{\partial u_{i}^{-}}{\partial x_{j}} v_{i} d x \\
& -\frac{1}{2} \int_{\Gamma}\left|\boldsymbol{u}^{-}\right|^{2} \boldsymbol{v} \cdot \boldsymbol{n} d S+\int_{\Gamma} \lambda\left(\boldsymbol{u}^{+}, p^{+}\right) \cdot \boldsymbol{v} d S \\
& =\int_{\Omega^{-}} \boldsymbol{f} \cdot \boldsymbol{v} d x
\end{aligned}
$$


which can be written in the abstract form

$$
a\left(\boldsymbol{u}^{-}, \boldsymbol{v}\right)+\left\langle\lambda\left(\boldsymbol{u}^{+}, p^{+}\right), \gamma_{0} \boldsymbol{v}\right\rangle=(\boldsymbol{f}, \boldsymbol{v})_{0, \Omega^{-}}, \quad \boldsymbol{v} \in \mathcal{V}\left(\Omega^{-}\right)
$$

where

$$
\begin{aligned}
& a(\boldsymbol{u}, \boldsymbol{v})=a_{0}(\boldsymbol{u}, \boldsymbol{v})+a_{1}(\boldsymbol{u}, \boldsymbol{u}, \boldsymbol{v})+a_{2}(\boldsymbol{u}, \boldsymbol{u}, \boldsymbol{v}), \\
& a_{0}(\boldsymbol{u}, \boldsymbol{v})=2 \nu \int_{\Omega^{-}} \sum_{i, j=1}^{3} D_{i j}(\boldsymbol{u}) D_{i j}(\boldsymbol{v}) d x \\
& a_{1}(\boldsymbol{u}, \boldsymbol{w}, \boldsymbol{v})=\int_{\Omega^{-}} \sum_{i, j=1}^{3} u_{j} \frac{\partial w_{i}}{\partial x_{j}} v_{i} d x, \\
& a_{2}(\boldsymbol{u}, \boldsymbol{w}, \boldsymbol{v})=-\frac{1}{2} \int_{\Gamma}(\boldsymbol{u} \cdot \boldsymbol{w})(\boldsymbol{v} \cdot \boldsymbol{n}) d S, \\
& \boldsymbol{u}=\left(u_{1}, u_{2}, u_{3}\right), \quad \boldsymbol{v}=\left(v_{1}, v_{2}, v_{3}\right), \quad \boldsymbol{w}=\left(w_{1}, w_{2}, w_{3}\right) \in C^{1}\left(\bar{\Omega}^{-}\right)
\end{aligned}
$$

(here $\boldsymbol{n}$ denotes the outer unit normal to $\partial \Omega^{-}$on $\Gamma$ ). These forms have the following properties:

Lemma 3.1 The forms $a_{0}, a_{1}, a_{2}$ are defined for all $\boldsymbol{u}, \boldsymbol{v}, \boldsymbol{w} \in \boldsymbol{H}^{1}\left(\Omega^{-}\right)$. The form $a_{0}$ is a continuous bilinear form on $\boldsymbol{H}^{1}\left(\Omega^{-}\right) \times \boldsymbol{H}^{1}\left(\Omega^{-}\right)$and $a_{1}, a_{2}$ are continuous trilinear forms on $\boldsymbol{H}^{1}\left(\Omega^{-}\right) \times \boldsymbol{H}^{1}\left(\Omega^{-}\right) \times \boldsymbol{H}^{1}\left(\Omega^{-}\right)$. If $\boldsymbol{f} \in \boldsymbol{L}^{2}\left(\Omega^{-}\right)$, then the mapping "v $\in \boldsymbol{H}^{1}\left(\Omega^{-}\right) \rightarrow(\boldsymbol{f}, \boldsymbol{v})_{0, \Omega^{-}} \in \mathbb{R}$ " is a continuous linear functional on $\boldsymbol{H}^{1}\left(\Omega^{-}\right)$. Provided $\lambda\left(\boldsymbol{u}^{+}, \boldsymbol{p}^{+}\right) \in \boldsymbol{H}^{-1 / 2}(\Gamma)$, the mapping " $\boldsymbol{v} \in \boldsymbol{H}^{1}\left(\Omega^{-}\right) \rightarrow\left\langle\lambda\left(\boldsymbol{u}^{+}, p^{+}\right), \gamma_{0} \boldsymbol{v}\right\rangle \in$ $\mathbb{R}^{\prime}$ is a continuous linear functional on $\boldsymbol{H}^{1}\left(\Omega^{-}\right)$.

Proof: The properties of $a_{0}, a_{1}$ and $(\boldsymbol{f}, \boldsymbol{v})_{0, \Omega^{-}}$are well-known (see, e.g., [6], [12], [25]). The properties of $a_{2}$ follow from the continuous imbedding of $\boldsymbol{H}^{1}\left(\Omega^{-}\right)$into $\boldsymbol{L}^{3}(\Gamma)$ (cf. [17], Par. 6.5). This and the Hölder inequality imply that for $\boldsymbol{u}, \boldsymbol{v}, \boldsymbol{w} \in$ $\boldsymbol{H}^{1}\left(\Omega^{-}\right)$,

$$
\begin{aligned}
\left|a_{2}(\boldsymbol{u}, \boldsymbol{v}, \boldsymbol{w})\right| & \leq \frac{1}{2}\left(\int_{\Gamma}\left|\gamma_{0} \boldsymbol{u}\right|^{3} d S\right)^{1 / 3}\left(\int_{\Gamma}\left|\gamma_{0} \boldsymbol{v}\right|^{3} d S\right)^{1 / 3}\left(\int_{\Gamma}\left|\gamma_{0} \boldsymbol{w}\right|^{3} d S\right)^{1 / 3} \\
& \leq c\|\boldsymbol{u}\|_{1, \Omega^{-}}\|\boldsymbol{v}\|_{1, \Omega^{-}}\|\boldsymbol{w}\|_{1, \Omega^{-}}
\end{aligned}
$$

with a constant $c$ independent of $\boldsymbol{u}, \boldsymbol{v}, \boldsymbol{w}$. The linearity of $a_{2}$ with respect to $\boldsymbol{u}, \boldsymbol{v}, \boldsymbol{w}$ is clear. The assertion on $\left\langle\lambda\left(\boldsymbol{u}^{+}, p^{+}\right), \gamma_{0} \boldsymbol{v}\right\rangle$ is an immediate consequence of the trace theorem for functions from $\boldsymbol{H}^{1}\left(\Omega^{-}\right)$.

In what follows, we denote by $\langle\cdot, \cdot\rangle_{\Omega^{-}}$the duality pairing between $\boldsymbol{V}\left(\Omega^{-}\right)$and the dual $\boldsymbol{V}\left(\Omega^{-}\right)^{*}$ of $\boldsymbol{V}\left(\Omega^{-}\right)$. We will assume that $\boldsymbol{f} \in \boldsymbol{V}\left(\Omega^{-}\right)^{*}$. 
The above considerations lead us to the formulation of a generalized problem in $\Omega^{-}$:

Given $\boldsymbol{f} \in \boldsymbol{V}\left(\Omega^{-}\right)^{*}, \lambda\left(\boldsymbol{u}^{+}, p^{+}\right) \in \boldsymbol{H}^{-1 / 2}(\Gamma)$, find $\boldsymbol{u}^{-}: \Omega^{-} \rightarrow \mathbb{R}^{3}$ satisfying the conditions

$$
\begin{aligned}
& \boldsymbol{u}^{-} \in \boldsymbol{V}\left(\Omega^{-}\right) \\
& a\left(\boldsymbol{u}^{-}, \boldsymbol{v}\right)+\left\langle\lambda\left(\boldsymbol{u}^{+}, p^{+}\right), \gamma_{0} \boldsymbol{v}\right\rangle=\langle\boldsymbol{f}, \boldsymbol{v}\rangle_{\Omega^{-}} \forall \boldsymbol{v} \in \boldsymbol{V}\left(\Omega^{-}\right)
\end{aligned}
$$

Remark 3.2 In virtue of [12], Chap. I, Theorem 2.3, to the solution $\boldsymbol{u}^{-}$of problem (3.4) there exists a function $p^{-} \in L^{2}\left(\Omega^{-}\right)$, unique up to an additive constant, such that $\boldsymbol{u}^{-}, p^{-}$satisfy (1.3b) in the sense of distributions in $\Omega^{-}$.

We proceed similarly in $\Omega^{+}$. Let us assume for now that $\boldsymbol{u}_{0}=\left.\boldsymbol{u}^{-}\right|_{\Gamma}$ is known and $\boldsymbol{u}_{0} \in \boldsymbol{H}_{0}^{1 / 2}(\Gamma)$. The constant function $\Omega^{+} \ni x \longmapsto \boldsymbol{u}_{\infty}$, is a solution of the Stokes system $(1.3 \mathrm{e}-\mathrm{f})$ with zero pressure and satisfies condition $(1.3 \mathrm{~g})$. Hence, for $\boldsymbol{u}^{+}, p^{+}$ from (1.3), the couple $\left(\boldsymbol{u}^{+}-\boldsymbol{u}_{\infty}, p^{+}\right)$is also a solution of $(1.3 \mathrm{e}-\mathrm{f})$ :

$$
\begin{aligned}
-\nu \Delta\left(\boldsymbol{u}^{+}-\boldsymbol{u}_{\infty}\right)+\nabla p^{+} & =0 \text { in } \Omega^{+}, \\
\operatorname{div}\left(\boldsymbol{u}^{+}-\boldsymbol{u}_{\infty}\right) & =0 \text { in } \Omega^{+} .
\end{aligned}
$$

Moreover,

$$
\int_{\Gamma} \boldsymbol{u}_{\infty} \cdot \boldsymbol{n} d S=0
$$

and, thus, $\boldsymbol{u}_{0}-\boldsymbol{u}_{\infty} \in \boldsymbol{H}_{0}^{1 / 2}(\Gamma)$. In virtue of Lemma 2.3, there exists a function $\boldsymbol{R}\left(\boldsymbol{u}_{0}-\boldsymbol{u}_{\infty}\right) \in \boldsymbol{W}\left(\Omega^{+}\right)$with $\gamma_{0} \boldsymbol{R}\left(\boldsymbol{u}_{0}-\boldsymbol{u}_{\infty}\right)=\left(\boldsymbol{u}_{0}-\boldsymbol{u}_{\infty}\right)$.

Multiplying (3.5a) by any $\boldsymbol{v} \in \mathcal{V}_{0}\left(\Omega^{+}\right)$, integrating over $\Omega^{+}$, using Green's theorem and the fact that $\operatorname{div} \boldsymbol{v}=0$ and $\operatorname{supp} \boldsymbol{v} \subset \Omega^{+}$is compact, we find that

$$
\int_{\Omega^{+}} \sum_{i, j=1}^{3} \frac{\partial\left(u_{i}^{+}-u_{\infty i}\right)}{\partial x_{j}} \frac{\partial v_{i}}{\partial x_{j}} d x=0, \quad \boldsymbol{v}=\left(v_{1}, v_{2}, v_{3}\right) \in \mathcal{V}_{0}\left(\Omega^{+}\right)
$$

This leads to the weak formulation of the exterior problem in $\Omega^{+}$: Given $\boldsymbol{u}_{0} \in \boldsymbol{H}_{0}^{1 / 2}(\Gamma)$, find $\boldsymbol{u}^{+}: \Omega^{+} \rightarrow \mathbb{R}^{3}$ such that

$$
\begin{gathered}
\left(\boldsymbol{u}^{+}-\boldsymbol{u}_{\infty}\right)-\boldsymbol{R}\left(\boldsymbol{u}_{0}-\boldsymbol{u}_{\infty}\right) \in \boldsymbol{V}_{0}\left(\Omega^{+}\right), \\
a^{+}\left(\boldsymbol{u}^{+}-\boldsymbol{u}_{\infty}, \boldsymbol{v}\right)=0 \quad \forall \boldsymbol{v} \in \boldsymbol{V}_{0}\left(\Omega^{+}\right)
\end{gathered}
$$

where

$$
a^{+}(\boldsymbol{z}, \boldsymbol{v})=\nu \int_{\Omega^{+}} \sum_{i, j=1}^{3} \frac{\partial z_{i}}{\partial x_{j}} \frac{\partial v_{i}}{\partial x_{j}} d x, \quad \boldsymbol{z}, \boldsymbol{v} \in \boldsymbol{W}^{1}\left(\Omega^{+}\right)
$$


Notice that (3.6a) implies that $\boldsymbol{u}^{+}-\boldsymbol{u}_{\infty} \in \boldsymbol{W}\left(\Omega^{+}\right)$and $\boldsymbol{u}^{+} \in \boldsymbol{H}^{1}(\tilde{\Omega})$ for any bounded domain $\tilde{\Omega} \subset \Omega^{+}$. Hence, $\gamma_{0} \boldsymbol{u}^{+}$is well defined and $\gamma_{0} \boldsymbol{u}^{+}=\gamma_{0}\left(\boldsymbol{u}^{+}-\boldsymbol{u}_{\infty}\right)+$ $\boldsymbol{u}_{\infty}=\boldsymbol{u}_{0}$

We can immediately formulate the following result:

Lemma $3.3 a^{+}$is a continuous, $\boldsymbol{W}^{1}\left(\Omega^{+}\right)$-elliptic bilinear form.

By [5], Proposition 3, page 155 and [10], Volume I, Section V, Lemma 1.1, it holds:

Lemma 3.4 If $\boldsymbol{u}^{+}$is a solution of Problem 3.6, then there exists a uniquely determined pressure $p^{+} \in L^{2}\left(\Omega^{+}\right)$such that

$$
a^{+}\left(\boldsymbol{u}^{+}-\boldsymbol{u}_{\infty}, \boldsymbol{v}\right)-\int_{\Omega^{+}} p^{+} \operatorname{div} \boldsymbol{v} d x=0 \quad \forall \boldsymbol{v} \in \boldsymbol{W}_{0}^{1}\left(\Omega^{+}\right)
$$

Moreover, there exists a constant $c_{2}>0$ independent of $\boldsymbol{u}^{+}$such that

$$
\left\|p^{+}\right\|_{0, \Omega+} \leq c_{2}\left\|\boldsymbol{u}^{+}-\boldsymbol{u}_{\infty}\right\|_{1, \Omega+}
$$

Now we introduce the following concept:

Definition 3.5 We define a weak solution of problem (1.3) as a couple of functions $\boldsymbol{u}^{-}: \Omega^{-} \rightarrow \mathbb{R}^{3}$ and $\boldsymbol{u}^{+}: \Omega^{+} \rightarrow \mathbb{R}^{3}$ satisfying conditions (3.4), (3.6) and

$$
\boldsymbol{u}_{0}=\gamma_{0} \boldsymbol{u}^{-}
$$

In what follows we will be concerned with the existence of a weak solution defined in 3.5. Simultaneously we will solve the problem how to specify the operator $\lambda\left(\boldsymbol{u}^{+}, p^{+}\right)$in the transmission condition (1.3i). Assuming for now that $\boldsymbol{u}^{-}$is known, we solve problem (3.6) with the boundary condition on $\Gamma$ given by (3.10). Let us suppose that the solution $\boldsymbol{u}^{+}$of (3.6) and the pressure $p^{+}$associated with $\boldsymbol{u}^{+}$by Lemma 3.4 allow us to express $\lambda\left(\boldsymbol{u}^{+}, p^{+}\right)$. Then, as we see, $\lambda\left(\boldsymbol{u}^{+}, p^{+}\right)$is a function of $\boldsymbol{u}_{0}=\gamma_{0} \boldsymbol{u}^{-}$:

$$
\lambda\left(\boldsymbol{u}^{+}, p^{+}\right)=\Lambda\left(\gamma_{0} \boldsymbol{u}^{-}\right)
$$

The continuous, linear mapping $\Lambda$ acting from $\boldsymbol{H}_{0}^{1 / 2}(\Gamma)$ into $\boldsymbol{H}^{-1 / 2}(\Gamma)$ via the solution of the exterior Stokes problem (3.6) is called the Steklov-Poincaré operator.

With the aid of the Steklov-Poincaré operator $\Lambda$, problem 3.5 is reformulated in the following way: 
Abstract generalized problem. Given $\Lambda: \boldsymbol{H}_{0}^{1 / 2}(\Gamma) \rightarrow \boldsymbol{H}^{-1 / 2}(\Gamma)$ and $\boldsymbol{f} \in$ $\boldsymbol{V}\left(\Omega^{-}\right)^{*}$, find $\boldsymbol{u}^{-}: \Omega^{-} \rightarrow \mathbb{R}^{3}$ such that

$$
\begin{aligned}
& \boldsymbol{u}^{-} \in \boldsymbol{V}\left(\Omega^{-}\right) \\
& a\left(\boldsymbol{u}^{-}, \boldsymbol{v}\right)+\left\langle\Lambda\left(\gamma_{0} \boldsymbol{u}^{-}\right), \gamma_{0} \boldsymbol{v}\right\rangle=\langle\boldsymbol{f}, \boldsymbol{v}\rangle_{\Omega^{-}} \forall \boldsymbol{v} \in \boldsymbol{V}\left(\Omega^{-}\right)
\end{aligned}
$$

The form $a$ is defined by (3.3).

Remark 3.6 The choice of the operator $\Lambda$ is governed by the same criteria as the choice of the mapping $\lambda$ (see Section 1 ). We require that

1) problem (3.12) has at least one solution,

2) operator $\Lambda$ is compatible with the exterior problem (3.6).

Of course, the choice of $\Lambda$ (and $\lambda$ ) should also be reasonable from the physical point of view.

In order to satisfy the first requirement, we discuss next the solvability of the abstract problem (3.12).

\section{Existence of a solution of the abstract problem}

First we establish some important properties of the forms $a_{1}$ and $a_{2}$ from (3.3).

Lemma 4.1 For all $\boldsymbol{u}, \boldsymbol{v} \in \boldsymbol{V}\left(\Omega^{-}\right)$we have

$$
a_{1}(\boldsymbol{u}, \boldsymbol{v}, \boldsymbol{v})=-a_{2}(\boldsymbol{v}, \boldsymbol{v}, \boldsymbol{u})
$$

Proof: Since $a_{1}, a_{2}$ are continuous trilinear forms on $\boldsymbol{V}\left(\Omega^{-}\right) \times \boldsymbol{V}\left(\Omega^{-}\right) \times \boldsymbol{V}\left(\Omega^{-}\right)$and $\mathcal{V}\left(\Omega^{-}\right)$is dense in $\boldsymbol{V}\left(\Omega^{-}\right)$(cf. (2.13) and Lemma 3.1), it is sufficient to prove (4.1) for $\boldsymbol{u}, \boldsymbol{v} \in \mathcal{V}\left(\Omega^{-}\right)$. For such functions Green's theorem implies that

$$
\begin{aligned}
a_{1}(\boldsymbol{u}, \boldsymbol{v}, \boldsymbol{v}) & =\int_{\Omega^{-}} \sum_{i, j=1}^{3} u_{j} \frac{\partial v_{i}}{\partial x_{j}} v_{i} d x=\frac{1}{2} \int_{\Omega^{-}} \sum_{i, j=1}^{3} u_{j} \frac{\partial v_{i}^{2}}{\partial x_{j}} d x \\
& =\frac{1}{2} \int_{\Gamma} \sum_{i, j=1}^{3} v_{i}^{2} u_{j} n_{j} d S-\frac{1}{2} \int_{\Omega^{-}} \sum_{i=1}^{3} v_{i}^{2} \sum_{j=1}^{3} \frac{\partial u_{j}}{\partial x_{j}} d x \\
& =-a_{2}(\boldsymbol{v}, \boldsymbol{v}, \boldsymbol{u}),
\end{aligned}
$$

since $\left.\boldsymbol{v}\right|_{\Gamma_{0}}=0$ and div $\boldsymbol{u}=0$ in $\Omega^{-}$. 
Corollary 4.2 For $\boldsymbol{u}, \boldsymbol{v}, \boldsymbol{w} \in \boldsymbol{V}\left(\Omega^{-}\right)$we have

$$
a_{1}(\boldsymbol{u}, \boldsymbol{v}, \boldsymbol{w})=-a_{1}(\boldsymbol{u}, \boldsymbol{w}, \boldsymbol{v})-a_{2}(\boldsymbol{v}+\boldsymbol{w}, \boldsymbol{v}+\boldsymbol{w}, \boldsymbol{u})+a_{2}(\boldsymbol{v}, \boldsymbol{v}, \boldsymbol{u})+a_{2}(\boldsymbol{w}, \boldsymbol{w}, \boldsymbol{u}) .
$$

Proof: From the trilinearity of $a_{1}$ it follows that $a_{1}(\boldsymbol{u}, \boldsymbol{v}+\boldsymbol{w}, \boldsymbol{v}+\boldsymbol{w})=a_{1}(\boldsymbol{u}, \boldsymbol{v}, \boldsymbol{v})+$ $a_{1}(\boldsymbol{u}, \boldsymbol{v}, \boldsymbol{w})+a_{1}(\boldsymbol{u}, \boldsymbol{w}, \boldsymbol{v})+a_{1}(\boldsymbol{u}, \boldsymbol{w}, \boldsymbol{w})$. Now, (4.1) immediately yields (4.2).

Lemma 4.3 Let us define the form

$$
b(\boldsymbol{u}, \boldsymbol{v}, \boldsymbol{w})=a_{1}(\boldsymbol{u}, \boldsymbol{v}, \boldsymbol{w})+a_{2}(\boldsymbol{u}, \boldsymbol{v}, \boldsymbol{w}), \boldsymbol{u}, \boldsymbol{v}, \boldsymbol{w} \in \boldsymbol{H}^{1}\left(\Omega^{-}\right) .
$$

Then it holds: If $\boldsymbol{u}, \boldsymbol{v}, \boldsymbol{u}^{n} \in \boldsymbol{V}\left(\Omega^{-}\right), \quad n=1,2, \ldots, \boldsymbol{u}^{n} \rightarrow \boldsymbol{u}$ weakly in $\boldsymbol{V}\left(\Omega^{-}\right)$as $n \rightarrow \infty$, then

$$
b\left(\boldsymbol{u}^{n}, \boldsymbol{u}^{n}, \boldsymbol{v}\right) \rightarrow b(\boldsymbol{u}, \boldsymbol{u}, \boldsymbol{v}) \text { as } n \rightarrow \infty
$$

Proof: The proof is a consequence of the compact imbeddings $H^{1}\left(\Omega^{-}\right) \hookrightarrow \hookrightarrow L^{2}\left(\Omega^{-}\right)$ and $H^{1}\left(\Omega^{-}\right) \hookrightarrow \hookrightarrow L^{3}(\Gamma)$ (cf. [17], Par. 5.8.1, 6.10.5]). Hence,

$$
\boldsymbol{u}^{n} \rightarrow \boldsymbol{u} \text { strongly in } \boldsymbol{L}^{2}\left(\Omega^{-}\right) \text {as } n \rightarrow \infty
$$

and

$$
\gamma_{0} \boldsymbol{u}^{n} \rightarrow \gamma_{0} \boldsymbol{u} \text { strongly in } \boldsymbol{L}^{3}(\Gamma) \text { as } n \rightarrow \infty \text {. }
$$

In virtue of (4.2) and (4.3),

$$
\begin{aligned}
b\left(\boldsymbol{u}^{n}, \boldsymbol{u}^{n}, \boldsymbol{v}\right)= & -a_{1}\left(\boldsymbol{u}^{n}, \boldsymbol{v}, \boldsymbol{u}^{n}\right)- \\
& -a_{2}\left(\boldsymbol{u}^{n}+\boldsymbol{v}, \boldsymbol{u}^{n}+\boldsymbol{v}, \boldsymbol{u}^{n}\right)+a_{2}\left(\boldsymbol{u}^{n}, \boldsymbol{u}^{n}, \boldsymbol{u}^{n}\right)+ \\
& +a_{2}\left(\boldsymbol{v}, \boldsymbol{v}, \boldsymbol{u}^{n}\right)+a_{2}\left(\boldsymbol{u}^{n}, \boldsymbol{u}^{n}, \boldsymbol{v}\right) .
\end{aligned}
$$

The convergence

$$
a_{1}\left(\boldsymbol{u}^{n}, \boldsymbol{v}, \boldsymbol{u}^{n}\right) \rightarrow a_{1}(\boldsymbol{u}, \boldsymbol{v}, \boldsymbol{u}) \text { as } n \rightarrow \infty
$$

can be proved in a standard way as, e.g., in [12], page 286 or [6], Lemma 8.4.16: If $\boldsymbol{v} \in \mathcal{V}\left(\Omega^{-}\right)$, then

$$
\begin{aligned}
\left|a_{1}\left(\boldsymbol{u}^{n}, \boldsymbol{v}, \boldsymbol{u}^{n}\right)-a_{1}(\boldsymbol{u}, \boldsymbol{v}, \boldsymbol{u})\right| & =\left|\int_{\Omega^{-}} \sum_{i, j=1}^{3}\left(u_{j}^{n} u_{i}^{n}-u_{j} u_{i}\right) \frac{\partial v_{i}}{\partial x_{j}} d x\right| \leq \\
& \leq c(\boldsymbol{v}) \int_{\Omega^{-}} \sum_{i, j=1}^{3}\left|\left(u_{j}^{n} u_{i}^{n}-u_{j} u_{i}\right)\right| d x \rightarrow 0
\end{aligned}
$$

due to (4.5). Then (4.8) holds for any $\boldsymbol{v} \in \boldsymbol{V}\left(\Omega^{-}\right)$as follows from the density of $\mathcal{V}\left(\Omega^{-}\right)$in $\boldsymbol{V}\left(\Omega^{-}\right)$, continuity of $a_{1}$ and boundedness of the sequence $\left\{\boldsymbol{u}^{n}\right\}$. 
Furthermore, as a consequence of (4.6) we find that

$$
\begin{array}{ll}
a_{2}\left(\boldsymbol{u}^{n}+\boldsymbol{v}, \boldsymbol{u}^{n}+\boldsymbol{v}, \boldsymbol{u}^{n}\right) & \rightarrow a_{2}(\boldsymbol{u}+\boldsymbol{v}, \boldsymbol{u}+\boldsymbol{v}, \boldsymbol{u}), \\
a_{2}\left(\boldsymbol{u}^{n}, \boldsymbol{u}^{n}, \boldsymbol{u}^{n}\right) & \rightarrow a_{2}(\boldsymbol{u}, \boldsymbol{u}, \boldsymbol{u}), \\
a_{2}\left(\boldsymbol{v}, \boldsymbol{v}, \boldsymbol{u}^{n}\right) & \rightarrow a_{2}(\boldsymbol{v}, \boldsymbol{v}, \boldsymbol{u}), \\
a_{2}\left(\boldsymbol{u}^{n}, \boldsymbol{u}^{n}, \boldsymbol{v}\right) & \rightarrow a_{2}(\boldsymbol{u}, \boldsymbol{u}, \boldsymbol{v}) .
\end{array}
$$

Let us establish, e.g., the last limit in (4.9). Using (3.3) and the Hölder inequality, we find that

$$
\begin{aligned}
& \left|a_{2}\left(\boldsymbol{u}^{n}, \boldsymbol{u}^{n}, \boldsymbol{v}\right)-a_{2}(\boldsymbol{u}, \boldsymbol{u}, \boldsymbol{v})\right| \\
& \leq\left|a_{2}\left(\boldsymbol{u}^{n}, \boldsymbol{u}^{n}, \boldsymbol{v}\right)-a_{2}\left(\boldsymbol{u}^{n}, \boldsymbol{u}, \boldsymbol{v}\right)\right|+\left|a_{2}\left(\boldsymbol{u}^{n}, \boldsymbol{u}, \boldsymbol{v}\right)-a_{2}(\boldsymbol{u}, \boldsymbol{u}, \boldsymbol{v})\right| \\
& =\frac{1}{2}\left|\int_{\Gamma}\left[\boldsymbol{u}^{n} \cdot\left(\boldsymbol{u}^{n}-\boldsymbol{u}\right)\right](\boldsymbol{v} \cdot \boldsymbol{n}) d S\right|+\frac{1}{2}\left|\int_{\Gamma}\left[\left(\boldsymbol{u}^{n}-\boldsymbol{u}\right) \cdot \boldsymbol{u}\right](\boldsymbol{v} \cdot \boldsymbol{n}) d S\right| \\
& \leq \frac{1}{2}\left(\int_{\Gamma}\left(\left|\boldsymbol{u}^{n}\right|^{3}+|\boldsymbol{u}|^{3}\right) d S\right)^{1 / 3}\left(\int_{\Gamma}\left|\boldsymbol{u}^{n}-\boldsymbol{u}\right|^{3} d S\right)^{1 / 3}\left(\int_{\Gamma}|\boldsymbol{v}|^{3} d S\right)^{1 / 3} \\
& \rightarrow 0
\end{aligned}
$$

for $n \rightarrow \infty$, as follows from (4.6) and the boundedness of the sequence $\left\{\gamma_{0} \boldsymbol{u}^{n}\right\}$ in $\boldsymbol{L}^{3}(\Gamma)$. The other limits in (4.9) can be proved in a similar way. Now (4.7) - (4.9) imply (4.4).

Now we are in position to prove the following existence result for the abstract, generalized problem (3.12).

Theorem 4.4 Let $\nu>0$ and $\boldsymbol{f} \in \boldsymbol{V}\left(\Omega^{-}\right)^{*}$ be given and let $\Lambda: \boldsymbol{H}_{0}^{1 / 2}(\Gamma) \rightarrow$ $\boldsymbol{H}^{-1 / 2}(\Gamma)$ be a weakly sequentially continuous and weakly noncoercive mapping, i.e.,

$$
\begin{aligned}
& \boldsymbol{z}^{n}, \boldsymbol{z} \in \boldsymbol{H}_{0}^{1 / 2}(\Gamma), \quad \boldsymbol{z}^{n} \rightarrow \boldsymbol{z} \text { weakly in } \boldsymbol{H}^{1 / 2}(\Gamma) \text { as } n \rightarrow \infty \\
\Rightarrow & \left\langle\Lambda\left(\boldsymbol{z}^{n}\right), \boldsymbol{w}\right\rangle \rightarrow\langle\Lambda(\boldsymbol{z}), \boldsymbol{w}\rangle \forall \boldsymbol{w} \in \boldsymbol{H}^{1 / 2} \text { as } n \rightarrow \infty,
\end{aligned}
$$

and there exist constants $c_{3} \in \mathbb{R}$ and $c_{4} \geq 0$ such that

$$
\langle\Lambda(\boldsymbol{z}), \boldsymbol{z}\rangle \geq c_{3}-c_{4}\|\boldsymbol{z}\|_{1 / 2, \Gamma} \forall \boldsymbol{z} \in \boldsymbol{H}_{0}^{1 / 2}(\Gamma),
$$

respectively. Then Problem (3.12) has at least one solution $\boldsymbol{u}^{-} \in \boldsymbol{V}\left(\Omega^{-}\right)$.

Proof: We proceed analogously as in [12], Theorem 1.2, page 280 or [6], Par. 8.4.20.

There exists a sequence $\left\{\boldsymbol{w}^{i}\right\}_{i=1}^{\infty} \subset \mathcal{V}\left(\Omega^{-}\right)$of linearly independent elements such that

$$
\boldsymbol{V}\left(\Omega^{-}\right)=\text {closure of } \bigcup_{k=1}^{\infty} \boldsymbol{X}_{k} \text { in } \boldsymbol{H}^{1}\left(\Omega^{-}\right)
$$


where

$$
\boldsymbol{X}_{k}=\left[\boldsymbol{w}^{1}, \ldots, \boldsymbol{w}^{k}\right]
$$

is the linear space spanned by the set $\left\{\boldsymbol{w}^{1}, \ldots, \boldsymbol{w}^{k}\right\} . \boldsymbol{X}_{k}$ can be considered as a finite-dimensional Hilbert space equipped with the scalar product

$$
((\boldsymbol{u}, \boldsymbol{v}))=\int_{\Omega^{-}} \sum_{i, j=1}^{3} \frac{\partial u_{i}}{\partial x_{j}} \frac{\partial v_{i}}{\partial x_{j}} d x
$$

defined in $\boldsymbol{V}\left(\Omega^{-}\right)$.

For any $k=1,2, \ldots$, let $\boldsymbol{u}^{k} \in \boldsymbol{X}_{k}$ satisfy

$$
a\left(\boldsymbol{u}^{k}, \boldsymbol{w}^{i}\right)+\left\langle\Lambda\left(\gamma_{0} \boldsymbol{u}^{k}\right), \gamma_{0} \boldsymbol{w}^{i}\right\rangle=\left\langle f, \boldsymbol{w}^{i}\right\rangle_{\Omega^{-}}, \quad i=1, \ldots, k
$$

Since

$$
\boldsymbol{u}^{k}=\sum_{j=1}^{k} \xi_{j}^{k} \boldsymbol{w}^{j}, \quad \xi_{j}^{k} \in \mathbb{R},
$$

conditions (4.15) represent a system of $k$ nonlinear algebraic equations with respect to the unknowns $\xi_{1}^{k}, \ldots, \xi_{k}^{k}$. First, let us prove the existence of the solution $\boldsymbol{u}^{k}$ of $(4.15)$.

By Lemma 3.4 and the trace theorem, for any fixed $\boldsymbol{u} \in \boldsymbol{X}_{k}$, the mapping "v $\in \boldsymbol{X}_{k} \rightarrow a(\boldsymbol{u}, \boldsymbol{v})+\left\langle\Lambda\left(\gamma_{0} \boldsymbol{u}\right), \gamma_{0} \boldsymbol{v}\right\rangle-\langle\boldsymbol{f}, \boldsymbol{v}\rangle_{\Omega^{-}} \in \mathbb{R}$ " is a continuous linear functional on $\boldsymbol{X}_{k}$. By the Riesz representation theorem, there exists $\boldsymbol{P}_{k}(\boldsymbol{u}) \in \boldsymbol{X}_{k}$ such that

$$
\left(\left(\boldsymbol{P}_{k}(\boldsymbol{u}), \boldsymbol{v}\right)\right)=a(\boldsymbol{u}, \boldsymbol{v})+\left\langle\Lambda\left(\gamma_{0} \boldsymbol{u}\right), \gamma_{0} \boldsymbol{v}\right\rangle-\langle\boldsymbol{f}, \boldsymbol{v}\rangle_{\Omega^{-}}, \quad \boldsymbol{u}, \boldsymbol{v} \in \boldsymbol{X}_{k} .
$$

Hence, $\boldsymbol{P}_{k}: \boldsymbol{X}_{k} \rightarrow \boldsymbol{X}_{k}$. From the properties of the form $a$ and operator $\Lambda$ it follows that $\boldsymbol{P}_{k}$ is continuous in $\boldsymbol{X}_{k}$. Further, by (3.3), Lemma 4.1 and (4.11), we have

$$
\begin{aligned}
\left(\left(\boldsymbol{P}_{k}(\boldsymbol{u}), \boldsymbol{u}\right)\right)= & a_{0}(\boldsymbol{u}, \boldsymbol{u})+a_{1}(\boldsymbol{u}, \boldsymbol{u}, \boldsymbol{u})+a_{2}(\boldsymbol{u}, \boldsymbol{u}, \boldsymbol{u}) \\
+ & \left\langle\Lambda\left(\gamma_{0} \boldsymbol{u}\right), \gamma_{0} \boldsymbol{u}\right\rangle-\langle\boldsymbol{f}, \boldsymbol{u}\rangle_{\Omega^{-}} \\
\geq & 2 \nu \int_{\Omega^{-}} \sum_{i, j=1}^{3}\left|D_{i j}(\boldsymbol{u})\right|^{2} d x+c_{3}-c_{4}\left\|\gamma_{0} \boldsymbol{u}\right\|_{1 / 2, \Gamma} \\
& -\|\boldsymbol{f}\|_{V_{\left(\Omega^{-}\right)}}\|\boldsymbol{u}\|_{1, \Omega^{-}}
\end{aligned}
$$

By the trace theorem, there exists $c_{5}>0$ such that

$$
\left\|\gamma_{0} \boldsymbol{u}\right\|_{1 / 2, \Gamma} \leq c_{5}\|\boldsymbol{u}\|_{1, \Omega^{-}}, \quad \forall \boldsymbol{u} \in \boldsymbol{H}^{1}\left(\Omega^{-}\right)
$$


In virtue of Korn's inequality ([19], Par. 6.5, Theorem 3.1 and Par. 7.2.2, Lemma 2.1 ), there exists $c_{6}>0$ such that

$$
\begin{aligned}
& \int_{\Omega^{-}} \sum_{i, j=1}^{3}\left|D_{i j}(\boldsymbol{u})\right|^{2} d x \geq c_{6} \nu\|\boldsymbol{u}\|_{1, \Omega^{-}}^{2}, \\
& \boldsymbol{u} \in\left\{\boldsymbol{v} \in \boldsymbol{H}^{1}\left(\Omega^{-}\right) ;\left.\boldsymbol{u}\right|_{\Gamma_{0}}=0\right\} \supset \boldsymbol{V}\left(\Omega^{-}\right) .
\end{aligned}
$$

From (4.18) - (4.20) we see that

$$
\left(\left(\boldsymbol{P}_{k}(\boldsymbol{u}), \boldsymbol{u}\right)\right) \geq 2 \nu c_{6} \nu\|\boldsymbol{u}\|_{1, \Omega^{-}}^{2}-c\|\boldsymbol{u}\|_{1, \Omega^{-}}+c_{3}, \quad \boldsymbol{u} \in \boldsymbol{X}_{k},
$$

with some constant $c \geq 0$. Hence, there exists $K>0$ such that $\left(\left(\boldsymbol{P}_{k}(\boldsymbol{u}), \boldsymbol{u}\right)\right) \geq 0$ for all $\boldsymbol{u} \in \boldsymbol{X}_{k}$ with $\|\boldsymbol{u}\|_{1, \Omega^{-}}=K$.

Now [6], Lemma 4.1.53 or [18], Chap. I, Par. 4.3, Lemma 4.3 imply that for each $k=1,2, \ldots$ there exists at least one solution $\boldsymbol{u}^{k} \in \boldsymbol{X}_{k}$ with $\left\|\boldsymbol{u}^{k}\right\|_{1, \Omega^{-}} \leq K$ of the equation $\boldsymbol{P}_{k}\left(\boldsymbol{u}^{k}\right)=0$, equivalent to (4.15).

Hence, we get a sequence $\left\{\boldsymbol{u}^{k}\right\}_{k=1}^{\infty}$ of solutions of (4.15), bounded in $\boldsymbol{V}\left(\Omega^{-}\right)$. Since the space $\boldsymbol{V}\left(\Omega^{-}\right)$is reflexive, there exists a subsequence (for simplicity again denoted by $\left.\left\{\boldsymbol{u}^{k}\right\}_{k=1}^{\infty}\right)$ and $\boldsymbol{u} \in \boldsymbol{V}\left(\Omega^{-}\right)$such that

$$
\begin{aligned}
& \boldsymbol{u}^{k} \rightarrow \boldsymbol{u} \text { weakly in } \boldsymbol{V}\left(\Omega^{-}\right), \\
& \gamma_{0} \boldsymbol{u}^{k} \rightarrow \gamma_{0} \boldsymbol{u} \text { weakly in } \boldsymbol{H}^{1 / 2}(\Gamma), \\
& \text { as } k \rightarrow \infty
\end{aligned}
$$

Lemmas 3.1 and 4.3, assumption (4.10) and identity (4.15) imply that

$$
a\left(\boldsymbol{u}, \boldsymbol{w}^{i}\right)+\left\langle\Lambda\left(\gamma_{0} \boldsymbol{u}\right), \gamma_{0} \boldsymbol{w}^{i}\right\rangle=\left\langle\boldsymbol{f}, \boldsymbol{w}^{i}\right\rangle_{\Omega^{-}}, \quad i=1, \ldots, k, k=1,2, \ldots
$$

In view of $(4.12)$, we immediately get $(3.12 \mathrm{~b})$, which we wanted to prove.

\section{On the exterior Stokes problem and the associ- ated Steklov-Poincaré operator}

We start with the solvability of problem (3.6).

Theorem 5.1 There exists exactly one solution $\boldsymbol{u}^{+}$of problem (3.6). This solution is independent of the choice of the function $\boldsymbol{R}\left(\boldsymbol{u}_{0}-\boldsymbol{u}_{\infty}\right)$ satisfying the conditions

$$
\boldsymbol{R}\left(\boldsymbol{u}_{0}-\boldsymbol{u}_{\infty}\right) \in \boldsymbol{W}\left(\Omega^{+}\right), \quad \gamma_{0} \boldsymbol{R}\left(\boldsymbol{u}_{0}-\boldsymbol{u}_{\infty}\right)=\boldsymbol{u}_{0}-\boldsymbol{u}_{\infty} \text { on } \Gamma .
$$


There exists a constant $c_{7}>0$ independent of $\boldsymbol{u}_{0}$ and $\boldsymbol{u}_{\infty}$ such that

$$
\left\|\boldsymbol{u}^{+}-\boldsymbol{u}_{\infty}\right\|_{1, \Omega^{+}} \leq c_{7}\left\|\boldsymbol{u}_{0}-\boldsymbol{u}_{\infty}\right\|_{1 / 2, \Gamma}
$$

\section{Proof:}

a) The solution of interest can be written in the form

$$
\boldsymbol{u}^{+}=\boldsymbol{z}^{+}+\boldsymbol{R}\left(\boldsymbol{u}_{0}-\boldsymbol{u}_{\infty}\right)+\boldsymbol{u}_{\infty} \text { with } \boldsymbol{z}^{+} \in \boldsymbol{V}_{0}\left(\Omega^{+}\right)
$$

and problem (3.6) is equivalent to finding $z \in V_{0}\left(\Omega^{+}\right)$satisfying the identity

$$
a^{+}\left(\boldsymbol{z}^{+}, \boldsymbol{v}\right)=-a^{+}\left(\boldsymbol{R}\left(\boldsymbol{u}_{0}-\boldsymbol{u}_{\infty}\right), \boldsymbol{v}\right) \forall \boldsymbol{v} \in \boldsymbol{V}_{0}\left(\Omega^{+}\right)
$$

The Lax-Milgram lemma immediately yields the existence and uniqueness of such a $z^{+}$.

b) Let us show that $\boldsymbol{u}^{+}$defined by (5.3) and (5.4) is independent of the choice of $\boldsymbol{R}\left(\boldsymbol{u}_{0}-\boldsymbol{u}_{\infty}\right)$ satisfying (5.1). Suppose we have $\boldsymbol{R}_{i}=\boldsymbol{R}_{i}\left(\boldsymbol{u}_{0}-\boldsymbol{u}_{\infty}\right), \quad i=1,2$, satisfying (5.1) and denote by $\boldsymbol{u}_{i}^{+}$the associated solutions of (3.6). Since $\gamma_{0}\left(\boldsymbol{R}_{1}-\right.$ $\left.\boldsymbol{R}_{2}\right)=0$ on $\Gamma$, we have $\boldsymbol{R}_{1}-\boldsymbol{R}_{2} \in \boldsymbol{V}_{0}\left(\Omega^{+}\right)$. Moreover, taking into account that $\boldsymbol{u}_{i}^{+}$ satisfy (3.6 a), we see that $\boldsymbol{u}_{1}^{+}-\boldsymbol{u}_{2}^{+} \in \boldsymbol{V}_{0}\left(\Omega^{+}\right)$. Subtracting $(3.6 \mathrm{~b})$ for $\boldsymbol{u}_{i}^{+}, i=1,2$, and substituting $\boldsymbol{v}:=\boldsymbol{u}_{1}^{+}-\boldsymbol{u}_{2}^{+}$, we obtain

$$
\left|\boldsymbol{u}_{1}^{+}-\boldsymbol{u}_{2}^{+}\right|_{1, \Omega^{+}}^{2}=a^{+}\left(\boldsymbol{u}_{1}^{+}-\boldsymbol{u}_{2}^{+}, \boldsymbol{u}_{1}^{+}-\boldsymbol{u}_{2}^{+}\right)=0
$$

which implies that $\boldsymbol{u}_{1}^{+}=\boldsymbol{u}_{2}^{+}$.

c) Finally we prove (5.2). By (5.4) with $\boldsymbol{v}:=\boldsymbol{z}^{+}$,

$$
\left|\boldsymbol{z}^{+}\right|_{1, \Omega^{+}}^{2}=-a^{+}\left(\boldsymbol{R}\left(\boldsymbol{u}_{0}-\boldsymbol{u}_{\infty}\right), \boldsymbol{z}^{+}\right) \leq\left|\boldsymbol{R}\left(\boldsymbol{u}_{0}-\boldsymbol{u}_{\infty}\right)\right|_{1, \Omega^{+}}\left|\boldsymbol{z}^{+}\right|_{1, \Omega^{+}} .
$$

Hence,

$$
\left|z^{+}\right|_{1, \Omega^{+}} \leq\left|\boldsymbol{R}\left(\boldsymbol{u}_{0}-\boldsymbol{u}_{\infty}\right)\right|_{1, \Omega^{+}}
$$

This, (5.3) and the equivalence of the norms $|\cdot|_{1, \Omega^{+}}$and $\|\cdot\|_{1, \Omega^{+}}$on $\boldsymbol{V}_{0}\left(\Omega^{+}\right)$imply that there exists a constant $c>0$ independent of $\boldsymbol{u}_{\infty}, \boldsymbol{u}_{0}$ such that

$$
\left\|\boldsymbol{u}^{+}-\boldsymbol{u}_{\infty}\right\|_{1, \Omega+} \leq\left\|\boldsymbol{z}^{+}\right\|_{1, \Omega^{+}}+\left\|\boldsymbol{R}\left(\boldsymbol{u}_{0}-\boldsymbol{u}_{\infty}\right)\right\|_{1, \Omega^{+}} \leq c\left\|\boldsymbol{R}\left(\boldsymbol{u}_{0}-\boldsymbol{u}_{\infty}\right)\right\|_{1, \Omega^{+}}
$$

If $\boldsymbol{w} \in \boldsymbol{V}_{0}\left(\Omega^{+}\right)$, then $\boldsymbol{R}\left(\boldsymbol{u}_{0}-\boldsymbol{u}_{\infty}\right)+\boldsymbol{w}$ satisfies (5.1). Replacing $\boldsymbol{R}\left(\boldsymbol{u}_{0}-\boldsymbol{u}_{\infty}\right)$ by $\boldsymbol{R}\left(\boldsymbol{u}_{0}-\boldsymbol{u}_{\infty}\right)+\boldsymbol{w}$ in (3.6), we obtain the same solution $\boldsymbol{u}^{+}$of (3.6), as follows from part b) of this proof. Hence, by (5.5), we have

$$
\left\|\boldsymbol{u}^{+}-\boldsymbol{u}_{\infty}\right\|_{1, \Omega^{+}} \leq c\left\|\boldsymbol{R}\left(\boldsymbol{u}_{0}-\boldsymbol{u}_{\infty}\right)+\boldsymbol{w}\right\|_{1, \Omega^{+}} \forall \boldsymbol{w} \in \boldsymbol{V}_{0}\left(\Omega^{+}\right)
$$


which means that

$$
\left\|\boldsymbol{u}^{+}-\boldsymbol{u}_{\infty}\right\|_{1, \Omega^{+}} \leq c \inf _{\boldsymbol{w} \in V_{0}\left(\Omega^{+}\right)}\left\|\boldsymbol{R}\left(\boldsymbol{u}_{0}-\boldsymbol{u}_{\infty}\right)+\boldsymbol{w}\right\|_{1, \Omega^{+}}
$$

Now, from (5.6) and Lemma 2.3 we immediately obtain (5.2) with $c_{7}:=c c_{1}$.

Corollary 5.2 Inequality (5.2) implies that

$$
\left\|\boldsymbol{u}^{+}-\boldsymbol{u}_{\infty}\right\|_{1, \Omega^{+}} \leq c_{7}\left(\left\|\boldsymbol{u}_{0}\right\|_{1 / 2, \Gamma}+\left\|\boldsymbol{u}_{\infty}\right\|_{1 / 2, \Gamma}\right)=c_{7}\left(\left\|\boldsymbol{u}_{0}\right\|_{1 / 2, \Gamma}+\left|\boldsymbol{u}_{\infty} \| \Gamma\right|^{1 / 2}\right),
$$

where $|\Gamma|$ is the surface measure of $\Gamma$.

Lemma 5.3 Let $\boldsymbol{u} \in \boldsymbol{H}_{\mathrm{loc}}^{1}\left(\Omega^{+}\right)$(i.e., $\boldsymbol{u} \in \boldsymbol{H}^{1}\left(\Omega^{*}\right)$ for any bounded domain $\Omega^{*} \subset$ $\left.\Omega^{+}\right)$and $\operatorname{div} \boldsymbol{u}=0$ in $\Omega^{+}$. Then for $i=1,2,3$

$$
\sum_{j=1}^{3} \frac{\partial}{\partial x_{j}}\left(\frac{\partial u_{i}}{\partial x_{j}}+\frac{\partial u_{j}}{\partial x_{i}}\right)=\Delta u_{i} \text { in } \Omega^{+}
$$

in the sense of distributions. This means that

$$
\int_{\Omega^{+}} \sum_{j=1}^{3}\left(\frac{\partial u_{i}}{\partial x_{j}}+\frac{\partial u_{j}}{\partial x_{i}}\right) \frac{\partial v}{\partial x_{j}} d x=\int_{\Omega^{+}} \sum_{j=1}^{3} \frac{\partial u_{i}}{\partial x_{j}} \frac{\partial v}{\partial x_{j}} d x \quad \forall v \in C_{0}^{\infty}\left(\Omega^{+}\right)
$$

Proof: Since $\boldsymbol{u} \in \boldsymbol{H}_{\mathrm{loc}}^{1}\left(\Omega^{+}\right)$, the definition of distribution derivatives implies that

$$
\frac{\partial}{\partial x_{j}}\left(\frac{\partial u_{i}}{\partial x_{j}}\right)=\frac{\partial^{2} u_{i}}{\partial x_{j}^{2}}, \frac{\partial}{\partial x_{j}}\left(\frac{\partial u_{j}}{\partial x_{i}}\right)=\frac{\partial^{2} u_{j}}{\partial x_{i} \partial x_{j}}=\frac{\partial^{2} u_{j}}{\partial x_{j} \partial x_{i}}=\frac{\partial}{\partial x_{i}}\left(\frac{\partial u_{j}}{\partial x_{j}}\right) \text {. }
$$

Thus, since div $\boldsymbol{u}=0$, we immediately obtain (5.8), which is equivalent to (5.9).

Corollary 5.4 Let $\boldsymbol{u} \in \boldsymbol{W}\left(\Omega^{+}\right)$. Then,

$$
2 \int_{\Omega^{+}} \sum_{i, j=1}^{3} D_{i j}(\boldsymbol{u}) D_{i j}(\boldsymbol{v}) d x=\int_{\Omega^{+}} \sum_{i, j=1}^{3} \frac{\partial u_{i}}{\partial x_{j}} \frac{\partial v_{i}}{\partial x_{j}} d x \quad \forall \boldsymbol{v} \in \boldsymbol{W}_{0}^{1}\left(\Omega^{+}\right) .
$$

Proof: Since both sides of (5.9) considered as functions of $v$ represent continuous linear functionals on $W_{0}^{1}\left(\Omega^{+}\right)$and $C_{0}^{\infty}\left(\Omega^{+}\right)$is dense in $W_{0}^{1}\left(\Omega^{+}\right),(5.9)$ holds for all $v \in W_{0}^{1}\left(\Omega^{+}\right)$. If $\boldsymbol{v}=\left(v_{1}, v_{2}, v_{3}\right) \in \boldsymbol{W}_{0}^{1}\left(\Omega^{+}\right)$, then substitution of $v:=v_{i}$ in $(5.9)$, summation over $i=1,2,3$ and a simple calculation yield (5.10).

Further, let us give an extension of the expression $\sigma_{n}\left(\boldsymbol{u}^{+}, p^{+}\right)$(defined in (1.5) for smooth $\boldsymbol{u}^{+}$and $p^{+}$) to the solution $\boldsymbol{u}^{+}$of problem (3.6) and the associated pressure $p^{+}$(see Lemma 3.4). For simplicity we write $\boldsymbol{u}, p$ instead of $\boldsymbol{u}^{+}$and $p^{+}$. 
Theorem 5.5 Let $\boldsymbol{u} \in \boldsymbol{H}_{\mathrm{loc}}^{1}\left(\Omega^{+}\right), \frac{\partial u_{i}}{\partial x_{j}}, p \in L^{2}\left(\Omega^{+}\right)(i, j=1,2,3)$ and let the distributions

$$
2 \nu \sum_{j=1}^{3} \frac{\partial}{\partial x_{j}} D_{i j}(\boldsymbol{u})-\frac{\partial p}{\partial x_{i}}, \quad i=1,2,3,
$$

satisfy the condition

$$
\left(1+|x|^{2}\right)^{1 / 2}\left(2 \nu \sum_{j=1}^{3} \frac{\partial}{\partial x_{j}} D_{i j}(\boldsymbol{u})-\frac{\partial p}{\partial x_{i}}\right) \in L^{2}\left(\Omega^{+}\right) .
$$

Then the formula

$$
\begin{aligned}
\left\langle\sigma_{\mathbf{n}}(\boldsymbol{u}, p), \boldsymbol{w}\right\rangle= & -2 \nu \int_{\Omega^{+}} \sum_{i, j=1}^{3} D_{i j}(\boldsymbol{u}) D_{i j}(\boldsymbol{v}) d x+\int_{\Omega^{+}} p \operatorname{div} \boldsymbol{v} d x- \\
& -\int_{\Omega^{+}} \sum_{i=1}^{3}\left(2 \nu \sum_{j=1}^{3} \frac{\partial}{\partial x_{j}} D_{i j}(\boldsymbol{u})-\frac{\partial p}{\partial x_{i}}\right) v_{i} d x
\end{aligned}
$$

for all $\boldsymbol{w} \in \boldsymbol{H}^{1 / 2}(\Gamma)$ and $\boldsymbol{v} \in \boldsymbol{W}^{1}\left(\Omega^{+}\right)$such that $\gamma_{0} \boldsymbol{v}=\boldsymbol{w}$ on $\Gamma$ determines an element from $\boldsymbol{H}^{-1 / 2}(\Gamma)$ denoted by $\sigma_{\mathbf{n}}(\boldsymbol{u}, p)$.

Proof:

1) Let us consider the right-hand side of (5.13) as a function of $\boldsymbol{v}$ and denote it by $\sigma(\boldsymbol{v})$. In view of the assumptions of the theorem, for $\boldsymbol{v} \in \boldsymbol{W}^{1}\left(\Omega^{+}\right)$, we find that

$$
\begin{aligned}
|\sigma(\boldsymbol{v})| \leq & \left|2 \nu \int_{\Omega^{+}} \sum_{i, j=1}^{3} D_{i j}(\boldsymbol{u}) D_{i j}(\boldsymbol{v}) d x\right|+\left|\int_{\Omega^{+}} p \operatorname{div} \boldsymbol{v} d x\right| \\
+ & \mid \int_{\Omega^{+}}\left[\left(1+|x|^{2}\right)^{1 / 2} \sum_{i=1}^{3}\left(2 \nu \sum_{j=1}^{3} \frac{\partial}{\partial x_{j}} D_{i j}(\boldsymbol{u})-\frac{\partial p}{\partial x_{i}}\right)\right] \cdot \\
& \cdot\left[\left(1+|x|^{2}\right)^{-1 / 2} v_{i}\right] d x \mid \leq \\
\leq & c|\boldsymbol{u}|_{1, \Omega^{+}}|\boldsymbol{v}|_{1, \Omega^{+}}+c\|p\|_{0, \Omega^{+}}|\boldsymbol{v}|_{1, \Omega^{+}} \\
+ & \left\{\sum_{i=1}^{3}\left\|\left(2 \nu \sum_{j=1}^{3} \frac{\partial}{\partial x_{j}} D_{i j}(\boldsymbol{u})-\frac{\partial p}{\partial x_{i}}\right)\left(1+|x|^{2}\right)^{1 / 2}\right\|_{0, \Omega^{+}}^{2}\right\}^{1 / 2} \\
& \cdot\left\{\sum_{i=1}^{3} \int_{\Omega^{+}} \frac{\left|v_{i}(x)\right|^{2}}{1+|x|^{2}} d x\right\}^{1 / 2} \leq \tilde{c}\|\boldsymbol{v}\|_{1, \Omega^{+}} .
\end{aligned}
$$

We see that $\sigma$ is a continuous linear functional on $\boldsymbol{W}^{1}\left(\Omega^{+}\right)$. 
2) For a given $\boldsymbol{w} \in \boldsymbol{H}^{1 / 2}(\Gamma), \sigma(\boldsymbol{v})$ is independent of the choice of $\boldsymbol{v} \in \boldsymbol{W}^{1}\left(\Omega^{+}\right)$such that $\gamma_{0} \boldsymbol{v}=\boldsymbol{w}$ on $\Gamma$. Actually, if $\boldsymbol{v}^{*}, \boldsymbol{v}^{* *} \in \boldsymbol{W}^{1}\left(\Omega^{+}\right)$and $\gamma_{0} \boldsymbol{v}^{*}=\gamma_{0} \boldsymbol{v}^{* *}=\boldsymbol{w}$, then $\boldsymbol{v}^{*}-\boldsymbol{v}^{* *} \in \boldsymbol{W}_{0}^{1}\left(\Omega^{+}\right)$. By the definition of the distributions (5.11), $\sigma(\boldsymbol{v})=0$ for all $\boldsymbol{v} \in C_{0}^{\infty}\left(\Omega^{+}\right)$. Since $C_{0}^{\infty}\left(\Omega^{+}\right)$is dense in $\boldsymbol{W}_{0}^{1}\left(\Omega^{+}\right), \sigma(\boldsymbol{v})=0$ for all $\boldsymbol{v} \in \boldsymbol{W}_{0}^{1}\left(\Omega^{+}\right)$ and, thus, $\sigma\left(\boldsymbol{v}^{*}\right)=\sigma\left(\boldsymbol{v}^{* *}\right)$.

3) The above results imply that the conditions

$$
\phi(\boldsymbol{w})=\sigma(\boldsymbol{v}), \quad \boldsymbol{w} \in \boldsymbol{H}^{1 / 2}(\Gamma), \boldsymbol{v} \in \boldsymbol{W}^{1}\left(\Omega^{+}\right), \quad \gamma_{0} \boldsymbol{v}=\boldsymbol{w}
$$

properly define a linear functional $\phi: \boldsymbol{H}^{1 / 2}(\Gamma) \rightarrow \mathbb{R}$. To show that $\phi$ is continuous, observe that by $(5.14),(5.15)$ and $(2.7)$,

$$
|\phi(\boldsymbol{w})|=\inf _{\substack{\boldsymbol{v} \in \boldsymbol{W}^{1}\left(\Omega^{+}\right) \\ \gamma_{0} \boldsymbol{v}_{=} \boldsymbol{w}}}|\sigma(\boldsymbol{v})| \leq \tilde{c} \inf _{\substack{\boldsymbol{v} \in \boldsymbol{W}^{1}\left(\Omega^{+}\right) \\ \gamma_{0} \boldsymbol{v}_{=} \boldsymbol{w}}}\|\boldsymbol{v}\|_{1, \Omega^{+}}=\tilde{c}\|\boldsymbol{w}\|_{1 / 2, \Gamma}
$$

This means that $\phi \in \boldsymbol{H}^{-1 / 2}(\Gamma)$. We shall denote it by $\sigma_{\mathbf{n}}(\boldsymbol{u}, p)$ and, hence, $\left\langle\sigma_{\mathbf{n}}(\boldsymbol{u}, p), \boldsymbol{w}\right\rangle=\phi(\boldsymbol{w})$ for all $\boldsymbol{w} \in \boldsymbol{H}^{1 / 2}(\Gamma)$.

Remark 5.6 If $\boldsymbol{u} \in C^{2}\left(\bar{\Omega}^{+}\right) \cap W^{1}\left(\Omega^{+}\right)$and $p \in C^{1}\left(\bar{\Omega}^{+}\right) \cap L^{2}\left(\Omega^{+}\right)$, then the functional $\sigma_{\mathbf{n}}(\boldsymbol{u}, p)$ from Theorem 5.5 coincides with $\sigma_{\mathbf{n}}(\boldsymbol{u}, p)$ defined by (1.5) and

$$
\left\langle\sigma_{\mathbf{n}}(\boldsymbol{u}, p), \boldsymbol{w}\right\rangle=\int_{\Gamma} \sigma_{\mathbf{n}}(\boldsymbol{u}, p) \boldsymbol{w} d S, \boldsymbol{w} \in \boldsymbol{H}^{1 / 2}(\Gamma) .
$$

The above considerations lead us to the natural choice of the mapping $\Lambda$ : $\boldsymbol{H}_{0}^{1 / 2}(\Gamma) \rightarrow \boldsymbol{H}^{-1 / 2}(\Gamma)$ in the abstract problem (3.12). For any given $\boldsymbol{u}_{0} \in \boldsymbol{H}_{0}^{1 / 2}(\Gamma)$ we construct the (unique) solution $\boldsymbol{u}^{+}$of problem (3.6) and the pressure $p^{+}$uniquely associated with $\boldsymbol{u}^{+}$according to Lemma 3.4. Then, in view of (3.8) and the inclusion $C_{0}^{\infty}\left(\Omega^{+}\right) \subset \boldsymbol{W}_{0}^{1}\left(\Omega^{+}\right)$

$$
2 \nu \sum_{j=1}^{3} \frac{\partial D_{i j}\left(\boldsymbol{u}^{+}\right)}{\partial x_{j}}-\frac{\partial p^{+}}{\partial x_{i}}=0 \text { in } \Omega^{+} \text {for } i=1,2,3,
$$

in the sense of distributions. By Theorem 5.5, we define $\sigma_{\mathbf{n}}\left(\boldsymbol{u}^{+}, p^{+}\right)$, which is the distributional normal component of the stress tensor (1.5). Let us now set

$$
\Lambda\left(\boldsymbol{u}_{0}\right)=\lambda\left(\boldsymbol{u}^{+}, p^{+}\right):=-\sigma_{\mathbf{n}}\left(\boldsymbol{u}^{+}, p^{+}\right) \in \boldsymbol{H}^{-1 / 2}(\Gamma) .
$$

With this choice of the operator $\Lambda$ we get a "weak form" of the transmission condition (1.3i) on $\Gamma$ corresponding in a natural way to the Stokes problem as an ansatz for the flow outside $\Gamma$. 
Definition 5.7 We call the mapping $\Lambda$ defined by (5.13) and (5.19) the SteklovPoincaré operator associated with the exterior Stokes problem (3.6).

Now we establish basic properties of the Steklov-Poincaré operator $\Lambda$ for the exterior Stokes problem.

\section{Theorem 5.8}

a) There exist constants $c_{3} \in \mathbb{R}$ and $c_{4} \geq 0$ such that

$$
\left\langle\Lambda\left(\boldsymbol{u}_{0}\right), \boldsymbol{u}_{0}\right\rangle \geq c_{3}-c_{4}\left\|\boldsymbol{u}_{0}\right\|_{1 / 2, \Gamma} \forall \boldsymbol{u}_{0} \in \boldsymbol{H}_{0}^{1 / 2}(\Gamma) .
$$

b) If $\boldsymbol{z}_{n}, \boldsymbol{z} \in \boldsymbol{H}_{0}^{1 / 2}(\Gamma), \boldsymbol{z}_{n} \rightarrow \boldsymbol{z}$ weakly in $\boldsymbol{H}^{1 / 2}(\Gamma)$ as $n \rightarrow \infty$, then

$$
\left\langle\Lambda\left(\boldsymbol{z}_{n}\right), \boldsymbol{w}\right\rangle \rightarrow\langle\Lambda(\boldsymbol{z}), \boldsymbol{w}\rangle \text { as } n \rightarrow \infty \forall \boldsymbol{w} \in \boldsymbol{H}^{1 / 2}(\Gamma)
$$

\section{Proof:}

a) For $\boldsymbol{u}_{0} \in \boldsymbol{H}_{0}^{1 / 2}(\Gamma)$ we denote by $\boldsymbol{u}^{+}$and $p^{+}$the solution of problem (3.6) and the pressure associated with $\boldsymbol{u}^{+}$according to Lemma 3.4. In view of (3.6) and (5.18), the assumptions of Theorem 5.5 are satisfied for $\boldsymbol{u}:=\boldsymbol{u}^{+}$and $p:=p^{+}$. Substituting in (5.13) $\boldsymbol{v}:=\boldsymbol{u}^{+}-\boldsymbol{u}_{\infty} \in \boldsymbol{W}^{1}\left(\Omega^{+}\right)$and using the relations $\operatorname{div} \boldsymbol{v}=0$ in $\Omega^{+}$, $\gamma_{0} \boldsymbol{v}=\boldsymbol{u}_{0}-\boldsymbol{u}_{\infty}$ on $\Gamma, D_{i j}(\boldsymbol{v})=D_{i j}\left(\boldsymbol{u}^{+}\right)$, we find that

$$
\left\langle\Lambda\left(\boldsymbol{u}_{0}\right), \boldsymbol{u}_{0}-\boldsymbol{u}_{\infty}\right\rangle=2 \nu \int_{\Omega^{+}} \sum_{i, j=1}^{3}\left|D_{i j}\left(\boldsymbol{u}^{+}\right)\right|^{2} d x \geq 0
$$

and

$$
\left\langle\Lambda\left(\boldsymbol{u}_{0}\right), \boldsymbol{u}_{0}\right\rangle \geq\left\langle\Lambda\left(\boldsymbol{u}_{0}\right), \boldsymbol{u}_{\infty}\right\rangle
$$

Since $\boldsymbol{u}_{\infty} \in \boldsymbol{H}_{0}^{1 / 2}(\Gamma)$, by Lemma 2.3 there exists $\boldsymbol{R} \boldsymbol{u}_{\infty} \in \boldsymbol{W}\left(\Omega^{+}\right)$such that $\boldsymbol{u}_{\infty}=$ $\gamma_{0} \boldsymbol{R} \boldsymbol{u}_{\infty}$ on $\Gamma$ and

$$
\inf _{\left.\boldsymbol{z} \in \boldsymbol{V}_{0(\Omega+}\right)}\left\|\boldsymbol{R} \boldsymbol{u}_{\infty}+\boldsymbol{z}\right\|_{1, \Omega^{+}} \leq c_{1}\left\|\boldsymbol{u}_{\infty}\right\|_{1 / 2, \Gamma}=c_{1}\left|\boldsymbol{u}_{\infty} \| \Gamma\right|^{1 / 2}
$$

With $\boldsymbol{z} \in \boldsymbol{V}_{0}\left(\Omega^{+}\right)$and $\boldsymbol{v}:=\boldsymbol{R} \boldsymbol{u}_{\infty}+\boldsymbol{z}$, we get from (5.13) and the Cauchy inequality

$$
\begin{aligned}
\left\langle\Lambda\left(\boldsymbol{u}_{0}\right), \boldsymbol{u}_{\infty}\right\rangle & =2 \nu \int_{\Omega^{+}} \sum_{i, j=1}^{3} D_{i j}\left(\boldsymbol{u}^{+}-\boldsymbol{u}_{\infty}\right) D_{i j}\left(\boldsymbol{R} \boldsymbol{u}_{\infty}+\boldsymbol{z}\right) d x \\
& \geq-2 \nu\left\|\boldsymbol{u}^{+}-\boldsymbol{u}_{\infty}\right\|_{1, \Omega^{+}}\left\|\boldsymbol{R} \boldsymbol{u}_{\infty}+\boldsymbol{z}\right\|_{1, \Omega^{+}}
\end{aligned}
$$

This, (5.22) and (5.7) imply that

$$
\begin{aligned}
\left\langle\Lambda\left(\boldsymbol{u}_{0}\right), \boldsymbol{u}_{\infty}\right\rangle & \geq-2 \nu\left\|\boldsymbol{u}^{+}-\boldsymbol{u}_{\infty}\right\|_{1, \Omega^{+}} \inf _{\left.\boldsymbol{z} \in \boldsymbol{V}_{0(\Omega+}\right)}\left\|\boldsymbol{R} \boldsymbol{u}_{\infty}+\boldsymbol{z}\right\|_{1, \Omega^{+}} \\
& \geq-2 \nu c_{1} c_{7}\left(\left\|\boldsymbol{u}_{0}\right\|_{1 / 2, \Gamma}+\left|\boldsymbol{u}_{\infty}\right||\Gamma|^{1 / 2}\right)\left|\boldsymbol{u}_{\infty} \| \Gamma\right|^{1 / 2}
\end{aligned}
$$


From here and (5.21) we immediately get (5.20).

b) Let $\hat{\Lambda}$ be the Steklov-Poincaré operator associated with the exterior Stokes problem (3.6), where $\boldsymbol{u}_{\infty}=0$. Then $\hat{\Lambda}$ is a continuous linear operator on $\boldsymbol{H}_{0}^{1 / 2}(\Gamma)$. To prove this, we consider $\boldsymbol{u}_{0}^{i} \in \boldsymbol{H}_{0}^{1 / 2}(\Gamma), \quad i=1,2$, and by $\boldsymbol{u}^{i+}, p^{i+}$ we denote the solution of (3.6) with $\boldsymbol{u}_{\infty}:=0$ and $\boldsymbol{u}_{0}:=\boldsymbol{u}_{0}^{i}$ and the associated pressures (cf. Lemma 3.4). Then, for $\alpha_{i} \in \mathbb{R}, \quad i=1,2$, the function $\tilde{\boldsymbol{u}}^{+}=\alpha_{1} \boldsymbol{u}^{1+}+\alpha_{2} \boldsymbol{u}^{2+}$ is the solution of (3.6), where $\boldsymbol{u}_{\infty}:=0$ and $\boldsymbol{u}_{0}:=\alpha_{1} \boldsymbol{u}_{0}^{1}+\alpha_{2} \boldsymbol{u}_{0}^{2}$. Moreover, $\tilde{p}^{+}:=\alpha_{1} p^{1+}+\alpha_{2} p^{2+}$ is the pressure associated with $\tilde{\boldsymbol{u}}^{+}$. From the definition of $\hat{\Lambda}$ (cf. (5.13) and (5.19)) we see that

$$
\hat{\Lambda}\left(\alpha_{1} \boldsymbol{u}_{0}^{1+}+\alpha_{2} \boldsymbol{u}_{0}^{2+}\right)=\alpha_{1} \hat{\Lambda}\left(\boldsymbol{u}_{0}^{1+}\right)+\alpha_{2} \hat{\Lambda}\left(\boldsymbol{u}_{0}^{2+}\right),
$$

which means that $\hat{\Lambda}$ is linear.

Further, we show that

$$
\left\|\hat{\Lambda}\left(\boldsymbol{u}_{0}\right)\right\|_{-1 / 2, \Gamma}:=\sup _{0 \neq \boldsymbol{w} \in \boldsymbol{H}^{1 / 2}(\Gamma)} \frac{\left\langle\hat{\Lambda}\left(\boldsymbol{u}_{0}\right), \boldsymbol{w}\right\rangle}{\|\boldsymbol{w}\|_{1 / 2, \Gamma}} \leq c_{8}\left\|\boldsymbol{u}_{0}\right\|_{1 / 2, \Gamma} \forall \boldsymbol{u}_{0} \in \boldsymbol{H}_{0}^{1 / 2}(\Gamma)
$$

with a constant $c_{8} \geq 0$ independent of $\boldsymbol{u}_{0}$. Let $\boldsymbol{u}^{+}$be the solution of problem (3.6) with $\boldsymbol{u}_{0} \in \boldsymbol{H}_{0}^{1 / 2}(\Gamma), \boldsymbol{u}_{\infty}=0$ and let $p^{+}$be the pressure associated with $\boldsymbol{u}^{+}$according to Lemma 3.4. Using (5.18), (5.19) and (2.7), similarly as in the proof of Theorem 5.5 (where we set $\boldsymbol{u}:=\boldsymbol{u}^{+}, p:=p^{+}$), for any $\boldsymbol{w} \in \boldsymbol{H}^{1 / 2}(\Gamma)$ we get

$$
\begin{aligned}
\left\langle\hat{\Lambda}\left(\boldsymbol{u}_{0}\right), \boldsymbol{w}\right\rangle & \leq c\left(\left\|\boldsymbol{u}^{+}\right\|_{1, \Omega^{+}}+\left\|p^{+}\right\|_{0, \Omega^{+}}\right) \inf _{\substack{\boldsymbol{v} \in W^{1}\left(\Omega^{+}\right) \\
\gamma_{0} \boldsymbol{v}_{=} \boldsymbol{w}}}\|\boldsymbol{v}\|_{1, \Omega^{+}}= \\
& =c\left(\left\|\boldsymbol{u}^{+}\right\|_{1, \Omega^{+}}+\left\|p^{+}\right\|_{0, \Omega^{+}}\right)\|\boldsymbol{w}\|_{1 / 2, \Gamma}
\end{aligned}
$$

For $\boldsymbol{u}_{\infty}=0$ from (5.24), (5.2) and (3.9) we obtain (5.23).

Now let us consider an arbitrary $\boldsymbol{u}_{\infty} \in \mathbb{R}^{3}$ and $\boldsymbol{u}_{0}^{i} \in \boldsymbol{H}_{0}^{1 / 2}(\Gamma), \quad i=1,2$. By $\boldsymbol{u}^{i+}$ we denote the solution of (3.6) and $p^{i+}$ the pressure associated with $\boldsymbol{u}^{i+}$ according to Lemma 3.4. Then $\boldsymbol{u}^{1+}-\boldsymbol{u}^{2+}$ is a solution of (3.6) with $\boldsymbol{u}_{\infty}:=0$ and $\boldsymbol{u}_{0}:=\boldsymbol{u}_{0}^{1}-\boldsymbol{u}_{0}^{2}$; $p^{1+}-p^{2+}$ is the corresponding pressure. From this, (5.13) and (5.19) we can see that

$$
\Lambda\left(\boldsymbol{u}_{0}^{1}\right)-\Lambda\left(\boldsymbol{u}_{0}^{2}\right)=\hat{\Lambda}\left(\boldsymbol{u}_{0}^{1}-\boldsymbol{u}_{0}^{2}\right)
$$

Let $\boldsymbol{w} \in \boldsymbol{H}^{1 / 2}(\Gamma), \quad \boldsymbol{z}_{n}, \boldsymbol{z} \in \boldsymbol{H}_{0}^{1 / 2}(\Gamma)$ and $\boldsymbol{z}_{n} \rightarrow \boldsymbol{z}$ weakly in $\boldsymbol{H}^{1 / 2}(\Gamma)$ as $n \rightarrow \infty$. Denoting by $\hat{\Lambda}^{*}$ the adjoint operator to $\hat{\Lambda}$ and using (5.25), we have $\hat{\Lambda}^{*} \boldsymbol{w} \in \boldsymbol{H}^{-1 / 2}(\Gamma)$ (cf., e.g., [27], Chap VII, Theorem I) and

$$
\left\langle\Lambda\left(\boldsymbol{z}_{n}\right)-\Lambda(\boldsymbol{z}), \boldsymbol{w}\right\rangle=\left\langle\hat{\Lambda}\left(\boldsymbol{z}_{n}-\boldsymbol{z}\right), \boldsymbol{w}\right\rangle=\left\langle\hat{\Lambda}^{*} \boldsymbol{w}, \boldsymbol{z}_{n}-\boldsymbol{z}\right\rangle \rightarrow 0 \text { as } n \rightarrow \infty,
$$

which we wanted to prove. 
Summarizing the results in Theorems 4.4 and 5.8, we immediately get the existence of a weak solution of the coupled problem (1.3).

Theorem 5.9 Let $\nu>0, \boldsymbol{f} \in \boldsymbol{V}\left(\Omega^{-}\right)^{*}$ and $\boldsymbol{u}_{\infty} \in \mathbb{R}^{3}$ be given. If we define $\lambda\left(\boldsymbol{u}^{+}, p^{+}\right)$from the transmission condition (1.3i) by (5.19), then the coupled problem (1.3) has at least one weak solution $\boldsymbol{u}^{-}, \boldsymbol{u}^{+}$, in the sense of Definition 3.5.

Remark 5.10 As follows from the above considerations, we have constructed the transmission operator $\Lambda$ so that the requirements from Remark 3.6 are satisfied. The numerical realization of $\Lambda$ can be done, for example, with the representation of the nonlocal boundary operator $\Lambda$ with the aid of boundary integral equations and the fast numerical realization by means of multiscale or multipole algorithms. This will allow the numerical solution of problem (1.3) by the coupling of the finite element method in $\Omega^{+}$with the boundary element method on $\Gamma$, successfully applied to a number of exterior problems (see, e.g., [2], [4], [7], [11], $[24],[26])$.

\section{Remarks on coupling of the Navier-Stokes prob- lem with an exterior potential flow}

Experimental experience indicates that viscous effects are concentrated in many cases in the so-called boundary layer, i.e., in the vicinity of walls which form the boundary of the region occupied by the fluid, whereas outside the boundary layer the flow field has an inviscid (and even irrotational) character. This was observed already by L. Prandtl who proposed in [20] to investigate the flow of a real viscous fluid with small viscosity via the use of an inviscid solution combined with the solution of the viscous flow in the boundary layer. This suggests the idea to describe the flow in the exterior domain $\Omega^{\prime}$ with the aid of the coupling of viscous flow in the interior domain $\Omega^{-}$(containing the boundary layer) with inviscid flow in $\Omega^{+}$. This approach was used e.g. in [21], where a simplified model problem of the coupling of a linear viscous Stokes problem in $\Omega^{-}$with a linear inviscid Stokes problem in $\Omega^{+}$is investigated. As we shall show now, however, the coupling of Navier-Stokes and potential flow is mathematically well-posed, but can lead nevertheless to some paradoxical situations, from the physical point of view.

We are concerned with the complete Navier-Stokes system in $\Omega^{-}$coupled with inviscid irrotational flow in the exterior domain $\Omega^{+}$. Since $\Omega^{+}$is simply connected in the sense of [6], Definition 2.1.18, for any velocity field $\boldsymbol{u}^{+} \in C^{1}\left(\Omega^{+}\right)$ satisfying the condition rot $\boldsymbol{u}^{+}=0$ in $\Omega^{+}$, there exists the velocity potential $\Phi \in$ $C^{2}\left(\Omega^{+}\right)$(see [6], Theorem 2.1.34):

$$
\nabla \Phi=\boldsymbol{u}^{+} \text {in } \Omega^{+}
$$


Then the continuity equation is equivalent to the potential equation

$$
\Delta \Phi=0 \text { in } \Omega^{+}
$$

The pressure $p^{+}$is determined by $\boldsymbol{u}^{+}$with the aid of Bernoulli's equation

$$
p^{+}+\frac{1}{2}\left|\boldsymbol{u}^{+}\right|^{2}=c:=p_{\infty}+\frac{1}{2}\left|\boldsymbol{u}_{\infty}\right|^{2} \text { in } \Omega^{+}
$$

where $p_{\infty}$ and $\boldsymbol{u}_{\infty}$ are the pressure and velocity of the homogeneous flow at $\infty$.

So, in $\Omega^{-}$we consider the viscous system $(1.3 \mathrm{~b}-\mathrm{c})$ with the boundary condition $(1.3 \mathrm{~d})$ and in $\Omega^{+}$we use equation $(6.2)$. We must specify the condition at $\infty$ (analogous to $(1.3 \mathrm{~g})$ ) and the transmission conditions on $\Gamma$ (analogous to $(1.3 \mathrm{~h}-\mathrm{i})$ ).

For a prescribed velocity $\boldsymbol{u}_{\infty}$ at $\infty$ we define the corresponding velocity potential

$$
\Phi_{\infty}(x)=\boldsymbol{u}_{\infty} \cdot x, \quad x \in \mathbb{R}^{3}
$$

and the condition at $\infty$ will be written as

$$
\lim _{|x| \rightarrow \infty}\left(\Phi(x)-\Phi_{\infty}(x)\right)=0
$$

In view of (6.1), condition (1.3h) reads $\boldsymbol{u}^{-}=\nabla \Phi$ on $\Gamma$. However, this overdetermines equation (6.2) and must be relaxed. Therefore, instead of (1.3h) we use the transmission condition

$$
\boldsymbol{u}^{-} \cdot \boldsymbol{n}=\boldsymbol{u}^{+} \cdot \boldsymbol{n}=\frac{\partial \Phi}{\partial \boldsymbol{n}} \text { on } Г
$$

$\left(\partial / \partial \boldsymbol{n}\right.$ is the derivative in the direction of the unit normal $\boldsymbol{n}$ to $\Gamma$ pointing from $\Omega^{-}$ into $\Omega^{+}$.)

In order to modify condition (1.3i), we take into account that the viscous part of the stress tensor vanishes for inviscid flow in $\Omega^{+}$. Further, system $(6.1)-(6.3)$ is equivalent to the inviscid system consisting of the Euler equations, continuity equation and the condition rot $\boldsymbol{u}^{+}=0$ of irrotational flow. The dynamic pressure $p+1 / 2|\boldsymbol{u}|^{2}$ plays the same role for the Euler equations as for the Navier-Stokes equations. This leads us to the transmission condition:

$$
-\left(p^{-}+\frac{1}{2}\left|\boldsymbol{u}^{-}\right|^{2}\right) \boldsymbol{n}+2 \nu \operatorname{ID}(\boldsymbol{u}) n=-\left(p^{+}+\frac{1}{2}\left|\boldsymbol{u}^{+}\right|^{2}\right) \boldsymbol{n} \text { on } \Gamma \text {. }
$$

Using here Bernoulli's equation (6.3), we obtain

$$
-\left(p^{-}+\frac{1}{2}\left|\boldsymbol{u}^{-}\right|^{2}\right) \boldsymbol{n}+2 \nu \operatorname{ID}(\boldsymbol{u}) \boldsymbol{n}+c \boldsymbol{n}=0 \text { on } \Gamma \text {. }
$$


On the basis of the above consideration, we arrive at the following classical formulation of the viscous-inviscid coupled problem: Given $\nu>0, \boldsymbol{f}, \boldsymbol{u}_{\infty}, p_{\infty}$, find $\boldsymbol{u}^{-} \in C^{2}\left(\bar{\Omega}^{-}\right), p^{-} \in C^{1}\left(\bar{\Omega}^{-}\right)$and $\Phi \in C^{2}\left(\bar{\Omega}^{+}\right)$satisfying (1.3b - d ), (6.2), (6.5), (6.6), (6.7), with $\Phi_{\infty}$ defined by (6.4) and $c$ from (6.3).

As we see, the problem for $\boldsymbol{u}^{-}$and $p^{-}$can be considered independently of the problem for $\Phi$. Having obtained $\boldsymbol{u}^{-}, p^{-}$as a solution of $(1.3 \mathrm{~b}-\mathrm{d}$ ) and (6.7), we solve the problem $(6.2),(6.5),(6.6)$ for $\Phi$. The weak formulation of the interior problem (1.3b - d) can be written in the form of the abstract problem (3.12), where $\Lambda\left(\boldsymbol{u}_{0}\right)=c \boldsymbol{n}$ for all $\boldsymbol{u}_{0} \in \boldsymbol{H}_{0}^{1 / 2}(\Gamma)$. If $\Gamma$ is Lipschitz-continuous, then $c \boldsymbol{n} \in \boldsymbol{L}^{\infty}(\Gamma) \subset$ $\boldsymbol{H}^{-1 / 2}(\Gamma)$. In virtue of Theorem 4.4, the viscous interior problem has at least one weak solution $\boldsymbol{u}^{-} \in \boldsymbol{V}\left(\Omega^{-}\right)$.

The weak formulation of the exterior problem (6.2), (6.5), (6.6) reads: Find $\Phi: \Omega^{+} \rightarrow \mathbb{R}$ satisfying the conditions

$$
\begin{aligned}
& \Phi-\Phi_{\infty} \in W^{1}\left(\Omega^{+}\right), \\
& \int_{\Omega^{+}} \nabla\left(\Phi-\Phi_{\infty}\right) \cdot \nabla v d x=\int_{\Gamma}\left(\boldsymbol{u}^{-}-\boldsymbol{u}_{\infty}\right) \cdot \boldsymbol{n} v d S \quad \forall v \in W^{1}\left(\Omega^{+}\right) .
\end{aligned}
$$

With the aid of the Lax-Milgram lemma follows the existence and uniqueness of the solution of problem (6.8).

In summary, the nonlinear coupled problem (1.3b - 1.3d), (6.7) and (6.2), (6.5), (6.6) admits at least one weak solution and is therefore well-posed from a mathematical point of view. However, essential physical features may have been lost by adopting exterior potential flow in the far field. More precisely, (6.7) implies that $\Lambda\left(\gamma_{0} \boldsymbol{u}^{-}\right)$in $(3.12 b)$ is given by

$$
\Lambda=c \boldsymbol{n}, \quad c=p_{\infty}+\frac{1}{2}\left|\boldsymbol{u}_{\infty}\right|^{2}
$$

and hence that for all $\boldsymbol{v} \in \boldsymbol{V}\left(\Omega^{-}\right)$

$$
\left\langle\Lambda\left(\gamma_{0} \boldsymbol{u}^{-}\right), \gamma_{0} \boldsymbol{v}\right\rangle=\left\langle c \boldsymbol{n}, \gamma_{0} \boldsymbol{v}\right\rangle=c(\nabla \cdot \boldsymbol{v}, \mathbf{1})_{\Omega^{-}}=0
$$

Therefore we deduce that $\boldsymbol{u}^{-}$satisfies $(1.3 \mathrm{~b}-1.3 \mathrm{~d})$ and the homogeneous Neumann boundary condition

$$
\sigma_{\mathbf{n}}\left(\boldsymbol{u}^{-}, p^{-}\right)-\frac{1}{2}\left|\boldsymbol{u}^{-}\right|^{2} \boldsymbol{n}=0 \text { on } \Gamma
$$

and, in particular, that $\boldsymbol{u}^{-}$is independent of $\boldsymbol{u}_{\infty}, p_{\infty}$. If, moreover, $\boldsymbol{f}=0$ in (1.3b), we get $\boldsymbol{u}^{-}=0$ and the exterior problem becomes potential flow around 
the artificial, impermeable (since $0=\boldsymbol{u}^{-} \cdot \boldsymbol{n}=\frac{\partial \Phi}{\partial \boldsymbol{n}}$ on $\Gamma$ ) obstacle $\Gamma$. Therefore the coupling of Navier-Stokes with exterior potential flow is pathological from a physical point of view and should not be used, even though it gives rise to a mathematically well-posed coupled problem.

\section{Conclusion}

Our above considerations clear up in some sense the problem of the choice of suitable boundary conditions on the artificial boundary $\Gamma$, when we decide to truncate problem (1.1) to a bounded domain $\Omega^{-}$. As we see, the general form of a suitable artificial boundary condition prescribed on $\Gamma$ for the interior problem in $\Omega^{-}$reads

$$
-\left(p^{-}+\frac{1}{2}\left|\boldsymbol{u}^{-}\right|^{2}\right) \boldsymbol{n}+2 \nu \operatorname{ID}\left(\boldsymbol{u}^{-}\right) \boldsymbol{n}+\Lambda\left(\boldsymbol{u}^{-}\right)=0 \text { on } \Gamma .
$$

The operator $\Lambda$ is defined according to the approximation of the flow outside $\Gamma$.

As we have shown in the case of exterior potential flow, care must be taken in the selection of the flow model in $\Omega^{+}$, since not every mathematically well-posed model gives rise to physically meaningful results.

A similar approach can also be used in the case of a channel flow when the problem in an infinite channel is truncated to one in a bounded channel separated from the unbounded parts by surfaces $\Gamma$. Then condition (7.1) can again be used. It involves various artificial boundary conditions proposed, e.g., in [3], [14]. According to Remark 3.6, the choice of $\Lambda$ should guarantee the existence of a solution of the interior problem in $\Omega^{-}$with the artificial boundary condition (7.1). In this paper we have proposed and analyzed two possibilities of the construction of the operator $\Lambda$ satisfying this requirement.

Acknowledgements. The research of M. Feistauer has been supported under the Grant No.201/94/1067 of the Czech Grant Agency. The work was initiated during a visit of C. Schwab to Charles University and completed during a visit of $\mathrm{M}$. Feistauer to the ETH Zürich. The authors gratefully acknowledge the support of their institutions. 


\section{References}

[1] C. Bègue, E. Conca, F. Murat, O. Pironneau: The Stokes and Navier-Stokes equations with boundary conditions involving pressure. Publications du Laboratoire d'Analyse Numérique, Université P. et M. Curie, Paris VI, R 88027 , Novembre 1988.

[2] H. Berger, G. Warnecke, W. Wendland: Analysis of a FEM/BEM coupling method for transonic flow computations. Universität Stuttgart, Mathematisches Institut A, Preprint 93-9, (1993).

[3] Ch. H. Bruneau, P. Fabrie: Effective downstream boundary conditions for incompressible Navier-Stokes equations. Int. J. Numer. Meth. Fluids, 19, (1994), 693-705.

[4] M. Costabel, E.P. Stephan: Coupling of finite element and boundary element methods for an elasto-plastic interface problem. SIAM J. Numer. Anal., 27, (1990), 1212-1226.

[5] R. Dautray, J.L. Lions: Mathematical Analysis and Numerical Methods for Science and Technology, Vol. 4, Springer Verlag Berlin, Heidelberg, New York, (1993).

[6] M. Feistauer: Mathematical Methods in Fluid Dynamics. Pitman Monographs and Surveys in Pure and Applied Mathematics 67, Longman Scientific \& Technical, Harlow, (1993).

[7] M. Feistauer, G.C. Hsiao, R.E. Kleinman, R. Tezaur: Analysis and numerical realization of coupled BEM and FEM for nonlinear exterior problems. In: Methoden und Verfahren der Mathematischen Physik, Band 40, "Inverse Scattering and Potential problems in Mathematical Physics", (R.E. Kleinman, R. Kress, E. Martensen, eds.), P. Lang, Frankfurt am Main etc., (1995), 47-73.

[8] M. Feistauer and C. Schwab: in preparation.

[9] J. Franci: Monotone operators. A survey directed to applications to differential equations. Appl. Math., 35, (1990), 257-301.

[10] G.P. Galdi: An Introduction to the Mathematical Theory of the Navier-Stokes Equations. Springer Tracts in Natural Philosophy, Volume 38, Springer Verlag, New York etc., (1994).

[11] G.N. Gatica, G.C. Hsiao: The coupling of boundary element and finite element methods for a nonlinear exterior boundary value problem. Numer. Math., 61, (1992), 171-214. 
[12] V. Girault, P.A. Raviart: Finite Element Methods for Navier-Stokes Equations. Springer Series in Computational Mathematics 5, Springer-Verlag, Berlin, etc., (1986).

[13] F.K. Hebeker, P. Wilde: On missing boundary conditions with unsteady incompressible Navier-Stokes flows. Preprint, IBM Scientific Center, Heidelberg, 1990.

[14] J.G. Heywood, R. Rannacher, S. Turek: Artificial boundaries and flux and pressure conditions for the incompressible Navier-Stokes equations. Int. J. Numer. Meth. Fluids, (1996), to appear.

[15] Li K.-T., He Y.-N.: The coupling of boundary integral and finite element methods for the Navier-Stokes equations in an exterior domain. J. Comput. Math., 7, (1989), 157-173.

[16] Li K.-T., He Y.-N.: Coupling method for the exterior stationary Navier-Stokes equations. Num. Meth. Partial Diff. Equations, 9, (1993), 35-49.

[17] A. Kufner, O. John, S. Fučík: Function Spaces. Academia, Praha, (1977).

[18] J.L. Lions: Quelques méthodes de résolution des problèmes aux limites non linéaires. Dunod, Paris, (1969).

[19] J. Nečas, J. Hlaváček: Mathematical Theory of Elastic and Elasto-Plastic Bodies: An introduction. Elsevier, Amsterdam-Oxford-New York, (1981).

[20] L. Prandtl: Über Flüssigkeitsbewegungen bei sehr kleiner Reibung. Verh. d. III. Int. Math. Kongr. Heidelberg 1904, Teubner, (1905), 484-494.

[21] A. Quarteroni, G. Sacchi Landriani, A. Valli: Coupling of viscous and inviscid Stokes equations with a domain decomposition method for finite elements. Dipartimento di Matematica, Università degli Studi Trento, Preprint UTM 287, Ottobre 1989.

[22] R. Salvi: On the existence of weak solutions of a non-linear mixed problem for the Navier-Stokes equations in a time dependent domain. J. Fac. Science, Univ. Tokyo, 32, (1985), 213-221.

[23] R.L. Sani, P.M. Gresho: Résumé and remarks on the open boundary condition minisymposium. Int. J. Numer. Meth. Fluids, 18, (1994), 983-1008.

[24] A. Sequeira: The coupling of boundary integral and finite element methods for the bidimensional exterior steady problems. Math. Meth. in the Appl. Sci., 5, (1983), 356-375.

[25] R. Temam: Navier-Stokes Equations. North-Holland, Amsterdam-New YorkOxford, (1977). 
[26] W.L. Wendland: On asymptotic error estimates for the combined BEM and FEM. In: Finite Element and Boundary Element Techniques from Mathematical and Engineering Point of View (E. Stein and W.L. Wendland, eds.), CISM Lecture Notes 30, Springer-Verlag, Wien-New York, (1988), 273-333 .

[27] K. Yosida: Functional Analysis. Springer-Verlag, New York, (1974).

[28] A. Zaretti: Soluzioni stazionarie di un problema non lineare per le equazioni di Navier-Stokes. Instituto Lombardo, Accademia di Scienze e Lettere, Estratto dai Rendiconti, Classe di Scienze (A), 106, (1972), 354-464. 